\title{
RISK MANAGEMENT MAGAZINE
}

\section{Rivista dell'Associazione Italiana Financial Industry Risk Managers}

Anno 12, numero 1

Gennaio - Aprile 2017

Poste Italiane - Spedizione in abbonamento postale - 70\% aut. DCB / Genova nr. 569 anno 2005

TESTATA INDIPENDENTE CHE NON PERCEPISCE CONTRIBUTI PUBBLICI (legge 250/1990)

\section{IN QUESTO NUMERO}

\begin{tabular}{|l|l|}
\hline \multicolumn{2}{|l|}{} \\
\hline $\mathbf{2}$ & $\begin{array}{l}\text { Editoriale } \\
\text { di Maurizio Vallino } \\
\text { prospettiva del Risk Manager } \\
\text { Il principio contabile IFRS 9 in banca: la } \\
\text { di Nicola Pasturi e Luca Giuseppe Paulicelli }\end{array}$ \\
\hline $\mathbf{2 7}$ & $\begin{array}{l}\text { Il digital risk officer e l'analisi dei rischi } \\
\text { finanziari nelle imprese della digital } \\
\text { transformation } \\
\text { di Fabrizio Carapellotti - Romina Potenziani }\end{array}$ \\
\hline $\begin{array}{l}\text { L'algoritmo Fuzzy C-Means clustering } \\
\text { come tecnica automatica per } \\
\text { l'individuazione di anomalie di mercato: un } \\
\text { caso studio } \\
\text { di Pier Giuseppe Giribone, Ottavio Caligaris, } \\
\text { Simone Fioribello }\end{array}$ \\
\hline
\end{tabular}

Risk Management Magazine

Anno 12, $n^{\circ} 1$ Gennaio - Aprile 2017

Direttore Responsabile:

Maurizio Vallino

Condirettore

Corrado Meglio

Consiglio scientifico

Giampaolo Gabbi (Direttore del Consiglio Scientifico), AnnaBottasso, Ruggero Bertelli, Ottavio Caligaris, Simona Cosma, Paola Ferretti, Andrea Giacomelli, Adele Grassi, Pier Giuseppe Giribone, Duccio Martelli, Anna Grazia Quaranta, Enzo Scannella, Cristiana Schena, Giuseppe Torluccio

Comitato di redazione

Emanuele Diquattro, Fausto Galmarini, Rossano Giuppa, Aldo Letizia, Paolo Palliola, Enzo Rocca, Fabio Salis

Vignettista: Silvano Gaggero

Proprietà, Redazione e Segreteria:

Associazione Italiana Financial Industry Risk Managers (AIFIRM), Via Sile 18, 20139 Milano

Registrazione del Tribunale di Milano $n^{\circ} 629$ del $10 / 9 / 2004$

ISSN 2283-7329

E-mail: segreteria@aifirm.it;

Tel. 3896946315

Stampa: Algraphy S.n.c. - Passo Ponte Carrega 62-62r 16141 Genova

Le opinioni espresse negli articoli impegnano unicamente la responsabilità dei rispettivi autori

SPEDIZIONE IN ABBONAMENTO POSTALE AI SOCI AIFIRM RESIDENTI IN ITALIA, IN REGOLA CON L'ISCRIZIONE 


\section{EDITORIALE}

Cari lettori,

con questo numero, il primo del 2017, si formalizza un percorso iniziato 12 anni fa.

Premesso che l'obiettivo della rivista è sempre stato quello di essere un punto di incontro e di contatto tra chi, in Italia, si occupa di Risk Management, qualche mese fa, con 1'aiuto di alcuni accademici, abbiamo iniziato un percorso, che si concluderà a fine anno, per trasformare la nostra rivista in un periodico scientifico accreditato da AIDEA.

In questo senso abbiamo lavorato sulla qualità del Consiglio Scientifico e istituito il doppio referaggio anonimo degli articoli.

Tale direzione intrapresa dalla rivista è coerente con la scelta effettuata da Aifirm di essere sempre più un motore di sviluppo della ricerca in ambito di risk management, sia attraverso la pubblicazione di Position Paper sia con il progetto UNI volto a scrivere le "norme" della nostra dottrina in ambito ISO9000.

Il passo successivo, che si concretizza con questo primo numero, attiene al cambio del nome per essere aperti non solo verso i soci Aifirm, che sono i proprietari della testata, ma anche verso l'intera comunità scientifica che si occupa quotidianamente di gestione del rischio.

Il nuovo nome si accorcia e diventa semplicemente "Risk Management Magazine!..... così come cambia anche la periodicità, ora quadrimestrale, per gestire i tempi più lunghi e articolati del doppio referaggio.

\section{Maurizio Vallino}

\section{Processo di referaggio degli articoli proposti per la pubblicazione}

Gli articoli che sono proposti alla rivista per la pubblicazione sono sottoposti in forma anonima a due successivi livelli di referaggio.

Il primo livello di referaggio (di ammissibilità) viene effettuato sull'articolo dai membri del Consiglio Scientifico che ne valutano la conguità ai temi trattati dalla rivista.

Il secondo livello di referaggio (di pubblicabilità) viene effettuato sull'articolo da due referee scelti all'interno del Consiglio Scientifico o all'esterno tra accademici, ricercatori, esperti della materia, che ne valutano il contenuto e forma. 


\section{Il principio contabile IFRS 9 in banca: la prospettiva del Risk Manager \\ di Nicola Pasturi ${ }^{1}$ e Luca Giuseppe Paulicelli ${ }^{2}$ \\ Le opinioni espresse sono proprie degli autori e non vincolano in alcun modo le Istituzioni di appartenenza.}

\section{Abstract}

As a result of the financial crisis of 2008, the IASB (International Accounting Standards Board) decided to revise their accounting standards for financial instruments to address perceived deficiencies which were believed to have contributed to the magnitude of the crisis. IFRS 9 is one of the most important answers to this purpose. It prescribes a shift to a new loan classification scheme on three classes (stage) and introduces a new model to estimate provisions based on the expected loss model instead of the incurred but not reported model since the introduction of the IAS39. The allowances are based on the expected losses from defaults over the next 12 months (stage 1) unless there is a significant increase in credit risk (stage 2). If there is a significant increase in credit risk, the allowance is measured as the present value of all credit losses projected for the instrument over its full lifetime. Given this scenario, Institutions need to introduce new models or to specialize their credit risk models (PD, LGD, CCF and satellite models) useful for managerial and/or for capital requirements purposes to reflect the new standards. The position paper of AIFIRM, for which a summary is given below, is aimed at analyzing specific IFRS 9 requirements for a suitable application of new regulation.

\section{Introduzione}

Dopo la crisi del 2008, la comunità degli enti regolatori ha generalmente riconosciuto che i meccanismi di assorbimento delle perdite disponibili all'epoca hanno fallito nel cogliere tempestivamente il deterioramento del credito dell'industria bancaria. Da questa premessa nasce il Principio Contabile IFRS 9 redatto dallo IASB che prevede l'introduzione di un modello di impairment basato sul concetto di expected loss (perdita attesa), che va a sostituire l'attuale modello incurred loss (perdite sostenute) dello IAS 39, valido per tutti gli strumenti finanziari non rilevati al Fair Value con contropartita a conto economico.

In tale contesto le perdite attese possono essere contabilizzate subito indipendentemente dalla presenza o meno di un trigger event, e le stime devono essere continuamente adeguate sulla base non solo di fatti e dati passati e presenti, ma anche di previsioni. Nella valutazione vanno tenute anche in considerazione le variazioni del rischio di credito della controparte che determinano la classificazione di crediti in tre livelli (o "stage") a cui corrispondono distinte metodologie delle perdite da rilevare (1 year expected loss o lifetime expected loss). In sostanza, le differenze con lo IAS 39 non potrebbero essere più significative.

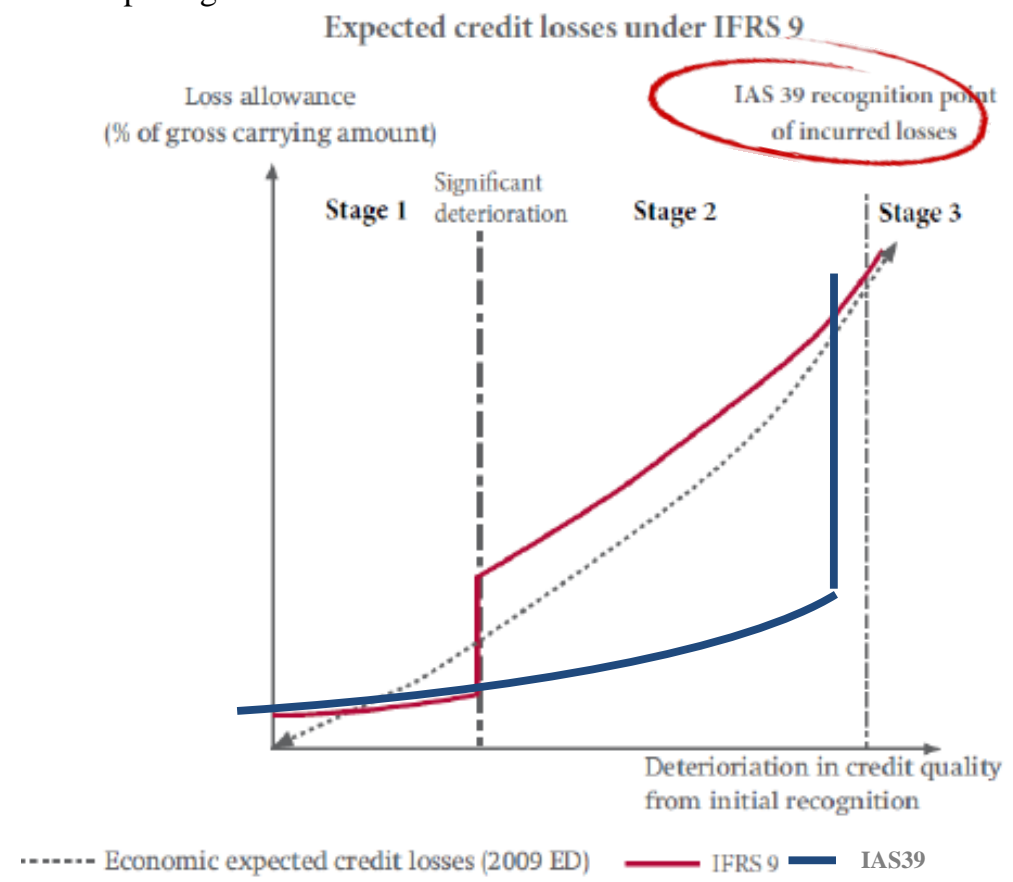

Figura 1 - Riconoscimento delle perdite su crediti IFRS 9 vs ED 2009 e IAS 39 (fonte: IASB Snapshot)

\footnotetext{
${ }^{1}$ Nicola Pasturi: Funzione Credit Portfolio Management- CRO Department - UBI Banca, PM Cantiere Modelli IFRS9.

${ }^{2}$ Luca Giuseppe Paulicelli: Responsabile Servizio Credit Portfolio Management \& Asset Quality Review - CRO Department - UBI Banca, Responsabile Cantiere Modelli IFRS9.
} 
In tale nuovo framework assume particolare rilevo la disponibilità di modelli interni per la misura delle perdite attese. È infatti necessario stimare le perdite future ( tecniche già adottate (per finalità gestionali o di vigilanza), modificandole per ottemperare ai requisiti previsti dal nuovo principio contabile. Valorizzando gli strumenti già esistenti, si tratta di affrontare lo sviluppo di ulteriori strumenti destinati alla migliore stima delle perdite attese pluriennali e dei processi volti a governare le future regole di passaggio dei crediti tra stadi diversi ("staging"), nonché i riflessi del nuovo sistema, sui processi di monitoraggio, sulle prassi di svalutazione e recupero del credito.

\section{L'allocazione di crediti tra stage}

L'impairment model previsto dall'IFRS 9 prevede la classificazione dei crediti in tre livelli (o "stage") a cui corrispondono distinte metodologie di calcolo delle perdite da rilevare.

Nel primo stage la perdita attesa è misurata entro un orizzonte temporale di un anno. Nel secondo stage (dove sono classificate le attività finanziarie che hanno subito un significativo incremento della rischiosità creditizia rispetto alla rilevazione iniziale), la perdita è misurata su un orizzonte temporale che copre la vita dello strumento sino a scadenza ("lifetime expected loss"). Nello stage 3 rientrano tutte quelle attività per cui l'aumento della rischiosità dal momento della rilevazione iniziale è stato così alto da far considerare le attività "impaired" (cioè per le quali si sono verificati eventi tali da incidere negativamente sui flussi di cassa stimati futuri come un mancato o ritardato pagamento).

Le indicazioni normative per l'allocazione dei crediti ai diversi "stage" previsti dal principio (c.d. attività di "staging" o "stage allocation") prevedono di identificare le variazioni significative del rischio di credito facendo riferimento ai seguenti aspetti principali:

- la variazione della probabilità di default rispetto alla prima rilevazione dell'attività finanziaria;

- la vita attesa dell'attività finanziaria;

- $\quad$ le informazioni forward looking che possono influenzare il rischio di credito.

Al fine di semplificare il processo di staging l'IFRS 9 propone due espedienti operativi ${ }^{3}$. Il primo è rappresentato dalla possibilità di evitare il passaggio in stage 2 se alla data di reporting lo strumento finanziario presenta un "basso rischio di credito" (cd. "low credit risk exemption"). La seconda semplificazione riguarda il passaggio da stage 1 a stage 2 in presenza di oltre 30 giorni di ritardo nei pagamenti (tale presunzione è per espressa indicazione normativa "rebuttable" cioè confutabile sulla base di evidenze ragionevoli e documentabili).

Nel lavoro viene presentato un possibile approccio alla stage allocation, articolato in 3 fasi:

1. analisi delle variazioni significative del merito creditizio con strumenti statistici

Il primo problema da affrontare sta nella selezione e nella calibrazione della PD all'erogazione, rispetto alla quale viene valutato l'aumento significativo della rischiosità:

- la PD alla nascita dell'operazione potrebbe essere non disponibile in quanto all'epoca i sistemi interni non erano ancora stati sviluppati, oppure hanno subito cambiamenti strutturali nel tempo che non la rendono confrontabile con la stessa misura alla data di riferimento. Per ovviare a tale problema, in sede di first time application, potrebbe risultare accettabile l'utilizzo della prima PD disponibile (anche se successiva all'origination), o ritenuta più affidabile.

- la PD alla nascita dell'operazione potrebbe non essere confrontabile con la PD alla reporting date per effetto di ricalibrazioni dei modelli. In tal caso, una correzione delle PD storiche dovrebbe essere applicata solo qualora il sistema di rating abbia prodotto una sistematica sottostima e/o sovrastima per un determinato periodo, oppure siano intervenute modifiche nella definizione di default e non per neutralizzare ricalibrazioni a seguito di un effettivo incremento della rischiosità delle controparti.

Successivamente, è necessario identificare la variabile target del motore statistico, per cui si pone la scelta nell'utilizzo tra PD a un anno, PD forward e PD lifetime. L'ultimo è l'approccio più coerente con il principio, in quanto viene considerato il rischio lungo l'intera vita residua, tuttavia altre motivazioni potrebbero condurre ad approfondire gli altri approcci (le misure ad un anno sono misure maggiormente conosciute e comprese dalle altre funzioni della banca ed, inoltre, le stime multi-periodali, essendo intrinsecamente più aleatorie, potrebbero comportare un notevole incremento del "rischio modello").

Infine, occorre selezionare la soglia che identifica il "significativo incremento del merito creditizio", per cui sono possibili metodologie basate sui rating ed altre basate su regressioni direttamente sulle PD:

${ }^{3}$ Sebbene l'uso di tali espedienti operativi sia previsto nell'IFRS 9, il Comitato di Basilea nelle sue Guidelines ha chiaramente affermato che l'uso di tali esenzioni da parte di banche di dimensione sovranazionale ("internationally active banks") dovrebbe essere limitato. 
- metodologia basata sulle matrici di migrazione, in cui vengono valutate le migrazioni tra classi di rating ed è finalizzata a mantenere un'attività in Stage 1 finché le sue possibili migrazioni cumulate non superano la soglia definita dal quantile.

- $\quad$ analisi di regressione direttamente sulle PD:

- regressione logistica

- regressione dei minimi quadrati

- regressione sui quantili

\section{2. valutazione andamentale}

Il secondo step del processo consiste nell'intercettare peggioramenti del rischio di credito non rilevati dal motore statistico, ed è legata al verificarsi di determinati trigger articolati nelle seguenti tipologie. Per esempio: classificazioni manageriali (watch list), 30 giorni di scaduto, forbearance (forbearance non performing, forbearance performing)

3. override manuale dei risultati (c.d. "IFRS 9 desk")

Infine, a valle del motore di calcolo statistico e della valutazione andamentale si potrebbe prevedere una struttura ad hoc ("IFRS 9 Desk") che si occupi di procedere d'iniziativa, per casistiche ben definite, alla riallocazione dei singoli rapporti in uno stage differente da quello determinato nelle due fasi precedenti (in analogia a quanto avviene spesso oggi con il rating).

\section{Il calcolo delle Expected Credit Losses: la stima di PD e LGD}

Nel nuovo principio le Expected Credit Losses vengono definite come una stima, pesata per le probabilità, del valore attuale di tutti i cash shortfall (intesi come differenza tra i flussi contrattuali e i flussi di cassa che la Banca stima di ricevere) attesi nel corso della vita del finanziamento (nel caso delle stime lifetime, lungo l'intera vita attesa dello strumento finanziario).

In tale impostazione le perdite attese vanno misurate in una modalità che rifletta:

- $\quad$ un ammontare obiettivo e pesato per le probabilità, che è determinato valutando un range di possibili risultati;

- $\quad$ il valore temporale del denaro;

- ogni ragionevole e supportabile informazione, disponibile senza eccessivi costi alla data di reporting, circa eventi passati, condizioni correnti e previsioni di condizioni economiche future.

Il principio specifica, poi, che le perdite attese vanno scontate alla reporting date utilizzando l'effective interest rate ("EIR") determinato alla data di recognition iniziale.

Alla luce di quanto sopra si possono così formulare le expected credit losses:

$$
E C L=\sum_{t=1}^{T} \frac{P D_{t^{*}} L G D_{t^{*} E A D_{t}}}{(1+E I R)^{t}}
$$

dove

- $\quad P D_{t}$ : probabilità di default nel periodo fra t e t-1,

- $L G D_{t}$ : loss given default nel periodo t,

- $E A D_{t}$ : exposure at default nel periodo t.

Se confrontato con la visione insita negli IAS vigenti, tale impostazione di perdita attesa [1] si avvicina senz'altro maggiormente a quello dei modelli IRB usati per la determinazione dei requisiti patrimoniali. Appare quindi ragionevole da più punti di vista partire dalla modellistica IRB - laddove presente - per rispondere al nuovo framework contabile in modo da cogliere le possibili sinergie e accrescere il grado di coerenza delle informazioni utilizzate nel proprio istituto di credito. A tale punto di partenza vanno apportati gli aggiustamenti per rendere i modelli aderenti al nuovo principio contabile, ovvero i parametri di rischio vanno resi:

- point-in-time, rispetto all'ottica through-the-cycle di Basilea;

- lifetime, rispetto all'ottica a 12 mesi di Basilea;

- $\quad$ inclusive di elementi forward looking.

Point in time

Nell'approccio cosidetto point in time (PIT), i parametri di rischio sono sensibili alle variazioni macroeconomiche di breve periodo, mentre in un approccio through the cycle (TTC) sono rimossi i fattori ciclici producendo stime del rischio di credito più stabili e meno volatili. 
La modalità più facile per gestire la conversione da una stima dei parametri effettuati in un'ottica TTC ad un'ottica PIT consiste nella scelta dell'orizzonte temporale di calibrazione, che va scelto in modo da cogliere la rischiosità corrente senza però compromettere la stabilità delle stime. In particolare, allungando l'orizzonte di calibrazione si otterranno parametri maggiormente TTC (adatti a fini regolamentari) oppure PIT (per finalità contabili/gestionali).

\section{Lifetime}

Come si è visto, per i crediti classificati in stage 2 vi è la necessità di quantificare la perdita attesa lungo l'intera classe di vita dei crediti, il che implica la stima di una struttura a termine dei parametri di rischio.

Partendo dalla PD, nel caso di portafogli con elevato numero di default (c.d. "high-default portfolios", quali ad esempio il Corporate ed il Retail), vi sono una serie di approcci caratterizzati da gradi di complessità differenti:

- catene di Markov: è una delle tecniche più utilizzate per la derivazione delle PD multiperiodali partendo da dati a un anno. Per derivare la struttura a termine è sufficiente stimare le possibilità di migrazione tra rating differenti e nello stato di default (stato assorbente): grazie alle ipotesi di omogeneità e di assenza di memoria è, quindi, possibile stimare la matrice di transizione sino all'anno $\alpha$ elevando all' $\alpha$-esima potenza la matrice a un anno. I vantaggi risiedono principalmente nell'agilità computazionale e nella possibilità di procedere sulla base di una limitata base informativa storica. Di contro non rispecchiano a pieno la logica dell'IFRS 9 poiché tale approccio non tiene conto dell'effetto sulle PD del tempo trascorso dall'erogazione ed, inoltre, dopo un certo periodo di tempo le probabilità tendono a distribuirsi in modo omogeneo intorno alla media di portafoglio (cd mean revertion).

- curve di vintage: tale metodo fa leva sull'esperienza interna per derivare l'andamento dei tassi di default nel medio-lungo periodo. L'obiettivo è costruire una matrice dei tassi di default suddivisa per anzianità ("vintage") di erogazione all'interno di ciascuna classe di rating, che riporti il livello dei tassi di default cumulati registrati su differenti orizzonti temporali. Il comportamento delle PD stimate (diversamente da quello mean reverting dell'approccio markoviano) risulta più conforme al reale ciclo di rischiosità del credito: i tassi di default, infatti, crescono inizialmente per poi decrescere all'avvicinarsi alla scadenza. Il limite di questa metodologia è la necessità di una serie storica ampia, con una profondità storica massima pari alla vita residua del portafoglio. In caso contrario è necessario introdurre metodologie per proiettare i tassi di default cumulati oltre l'ultimo periodo osservato.

- approcci ibridi "Markov per vintage": per superare i limiti strutturali dei due modelli sopra esposti è ipotizzabile l'utilizzo di approcci ibridi. Ovvero, data la maggior solidità delle curve vintage sulle scadenze brevi, appare ragionevole prevedere questo approccio come primo step. Una volta raggiunta la profondità massima, pari al vintage più vecchio, si proseguirà con il metodo delle catene di Markov.

- $\quad$ l'interpolazione dei default storici con funzioni di sopravvivenza: al fine di superare il vincolo che lega la stima delle PD lifetime alla massima profondità storica campionaria è possibile utilizzare un metodo statistico di stima della distribuzione probabilistica (ad esempio Weibull o lognormale) sulla base dei tassi di default empirici cumulati. In tal modo, una volta ottenuta la struttura a termine tramite l'interpolazione dei dati osservati, sarà possibile generare proiezioni degli anni futuri. Per applicare questo tipo di metodo è tuttavia essenziale disporre di un campione storico ampio, ed inoltre tra gli effetti indesiderati dell'interpolazione vi è la possibilità che le curve di PD cumulate delle diverse classi di rating si intersechino tra loro generando un fenomeno potenzialmente contro intuitivo a fronte del quale è necessario intervenire su base empirica.

Per i portafogli caratterizzati da un basso numero di default (c.d. "low-default portfolios", quali governi, banche centrali, compagnie di assicurazione, large corporate, ecc.) un approccio comunemente utilizzato è legato a modelli di tipo "shadow rating", basati su regressioni dove la variabile dipendente è costituita dalla PD associata ai rating che le controparti ottengono da una o più agenzie specializzate. La struttura a termine delle PD può, poi, essere ricavata dalla statistiche pubblicate dalle agenzie di rating.

In merito alla LGD, invece, occorre distinguere tra prodotti in cui la stima dei recuperi avviene tramite approcci di tipo "workout" perché in assenza di un mercato secondario (crediti a revoca, finanziamenti a scadenza e mutui ipotecari) ed il portafoglio titoli per cui è possibile utilizzare approcci che tengano conto dei dati di mercato disponibili.

Posta la formulazione standard della $\mathrm{LGD}^{4}$ :

${ }^{4}$ Non sempre i modelli a disposizione tengono conto di recovery rate relativi alla sola componente unsecured (per la componente secured si assume recupero pari al 100\%); è probabile che alcuni istituti adottino RR sensibili alla presenza/assenza delle garanzie ritenute significative in sede di stima o meglio ancora sensibili al relativo LTV. In questo caso è possibile che la formula adottata possa risultare differente e meno reattiva alla dinamica attesa del valore del collateral, anche in presenza di fasce di LTV che, per costruzione, non sono tendenzialmente numerose. 


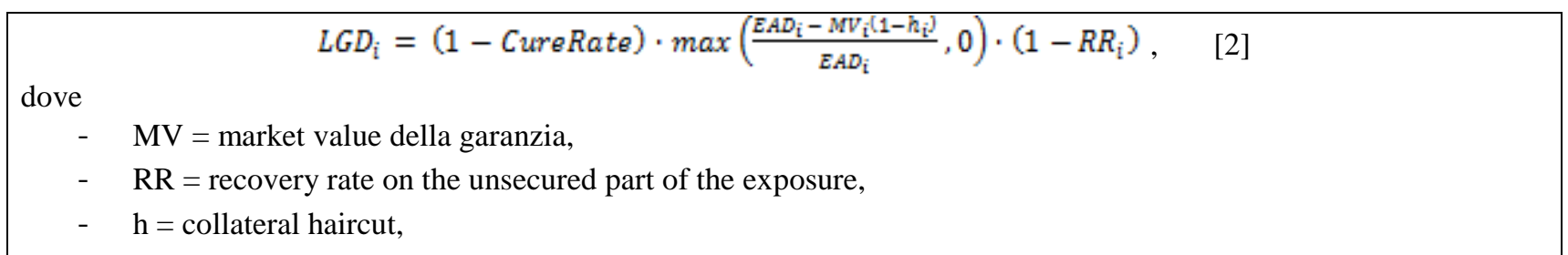

la stima di una struttura a termine va a considerare l'evoluzione del quoziente "loan to value" nel tempo in funzione della dinamica attesa del collateral: infatti, in particolare per i crediti garantiti da immobili (ma non esclusivamente), al ridursi del debito residuo aumenta, ceteris paribus, il tasso di copertura della garanzia (si riduce, cioè, il quoziente "loan to value") sulla base del piano di ammortamento contrattuale e di eventuali oscillazioni nel valore del cespite. Ciò conduce, di norma, ad una progressiva diminuzione delle LGD, spesso sugli orizzonti temporali caratterizzati da un aumento delle PD marginali.

\section{Forward looking}

Uno dei cambiamenti più significativi rispetto allo IAS 39 riguarda l'impiego non solo di informazioni storiche (ad esempio le informazioni circa le perdite passate su crediti), ricalibrate per incorporare gli effetti delle condizioni correnti, ma anche di previsioni future aventi un grado di giudizio e di accuratezza che dipende dalla disponibilità, dal dettaglio e dalla profondità dei dati stessi.

Le informazioni previsionali entrano già in taluni processi della banca (ICAAP/ILAAP, budgeting e forecasting, e nelle simulazioni ad hoc richieste dalle Autorità di Vigilanza), dove spesso viene posto l'accento sull'identificazione di scenari di stress. L'IFRS 9, invece, richiede la stima della perdita attesa sulla base di scenari "non distorti": l'obiettivo non è quello di pesare un "worst-case scenario" e un "best-case scenario", bensì quello di avere le migliori stime di perdita attesa seguendo criteri di "best estimate".

Alla luce di quanto richiesto dal principio è necessario affrontare i seguenti aspetti:

- $\quad$ le fonti dati utilizzate, il processo di selezione dei fattori macroeconomici rilevanti per le finalità previsionali e il loro legame con i differenti portafogli. Su questo punto si pone la scelta se fare leva su fonti informative pubbliche indipendenti e riconosciute (es. OECD, Eurostat, IMF, World Bank, Istat ...), oppure se utilizzare previsioni prodotte da una struttura di research interna.

- la natura del processo di generazione degli scenari macroeconomici, la scelta della loro numerosità, della profondità, la frequenza e profondità di aggiornamento degli stessi. È importante garantire coerenza e omogeneità di approccio tra $\mathrm{i}$ vari processi bancari che fanno leva su modelli che incorporano previsioni macroeconomiche.

- come tali scenari siano utilizzati per determinare la perdita attesa media ponderata per la probabilità di accadimento. In particolare, esistono diversi metodi per effettuare il collegamento tra gli scenari macroeconomici e la perdita attesa. I principali:

- calcolo dei parametri di rischio sulla base di una media ponderata dei parametri generati con diversi scenari;

- combinazione degli scenari macroeconomici possibili in un singolo scenario, da cui derivare i parametri di rischio;

- calcolo della perdita attesa con riferimento ad uno scenario baseline e la successiva correzione con un add-on che rifletta la non linearità della relazione tra scenari e perdite attese;

○ utilizzo di parametri di rischio non condizionati.

Infine, nel paper viene approfondito il tema della stima dei c.d. modelli satellite e della loro applicazione alla proiezione multiperiodale dei parametri di rischio. I modelli satellite sono modelli econometrici utilizzati per identificare e stimare la relazione tra le variabili utilizzate per la quantificazione dei rischi e un set di grandezze macroeconomiche e finanziarie suscettibili di influenzarne la dinamica. Essi sono tipicamente e ampiamente utilizzati nelle banche, nell'ambito di importati processi di pianificazione e risk management, tra cui la redazione del resoconto ICAAP e l'esecuzione degli stress test regolamentari. Lo sviluppo e la successiva applicazione di un modello di questo tipo comportano le seguenti fasi:

- identificazione della variabile target;

- $\quad$ analisi dei fattori macroeconomici e definizione di una long list;

- specificazione e stima dei possibili modelli e selezione di quello che meglio si adatta al comportamento delle serie storico e al loro significato economico;

- previsione della variabile dipendente. 


\section{Banche e portafogli trattati con modelli standard}

Finora è stato trattato il caso in cui sia disponibile una modellistica su cui far leva applicando le specificità richieste dall'IFRS 9. Nel caso di banche, o di singoli portafogli, per i quali non sono disponibili modelli sofisticati (per i quali il capitale di vigilanza viene calcolato secondo l'approccio "Standard") è possibile adottare delle semplificazioni nell'implementazione del principio.

Una prima alternativa, in questo caso, è fare affidamento su soluzioni esterne:

- modelli consortili: ricorso a centri consortili di servizi informatici (Cedacri, CSE, SEC, ecc.) cui spesso sono demandati anche lo sviluppo e la messa in opera di modelli gestionali.

- modelli forniti da società specializzate: sviluppati per singola banca e costruiti a partire unicamente dai sui dati. Come per i modelli consortili, vengono sfruttate ed estese ai modelli IFRS 9 le esperienze maturate soprattutto nell'ambito dei modelli gestionali di rating.

- modelli mutuati da prescrizioni della normativa di Vigilanza: ad esempio si riporta la circolare della Banca d'Italia n. 284 del 18 giugno 2013 ("Istruzioni per la compilazione delle segnalazioni delle perdite storicamente registrate sulle posizioni in default"), che si lega ad un archivio storico delle perdite coerente con la necessità di determinare le perdite attese su orizzonti temporali non limitati ai 12 mesi.

Oppure, la banca può approcciare lo sviluppo di modelli interni semplificati. Ad esempio:

- stage allocation: utilizzare un approccio basato su un opportuno set di indicatori quali-quantitativi della qualità creditizia, quali ad esempio la soglia dei 30 giorni di scaduto, la forbearance, indicatori qualitativi esterni (informazioni Centrale Rischi), watchlist e early warning system ed opinioni di esperti.

- PD: utilizzare l'applicazione di tassi di decadimento interni o esterni per i singoli portafogli in luogo della PD (eventualmente ricondotti a matrici di portafogli AIRB esistenti ed assimilabili per caratteristiche di rischiosità) e utilizzo di catene di Markov per estendere l'orizzonte previsivo.

- LGD: l'approccio probabilmente più evoluto potrebbe consistere nell'adeguare il modello della vigilanza (circ. 284 Bankit) gestendo le serie storiche coerentemente con i dettami del nuovo Principio.

\section{La First Time Application e gli impatti patrimoniali}

Dopo aver approfondito lo sviluppo dei modelli IRFS 9, si affrontano gli impatti attesi che avranno le nuove rettifiche calcolate secondo il nuovo modello di impairment descritto. Difatti, le rettifiche di valore su crediti sono un elemento chiave poiché hanno un significativo impatto sia sul Conto Economico che sul Capitale Regolamentare. Quindi, sia gli Accounting Standard Setters che i Regulators sono interessati alla loro definizione, seppur da una diversa prospettiva:

- report contabili: informazioni utili ad una vasta serie di soggetti che hanno una posizione neutrale alle perdite attese;

- vigilanza: ridurre livello di rischio per depositanti e mantenere una certa stabilità finanziaria ed un livello di Loan Loss Provisions che sia il più conservativo possibile.

Diviene, quindi cruciale analizzare la relazione tra il mondo contabile e quello prudenziale e come verrà a modificarsi con l'IFRS 9. Secondo il framework di Basilea, le rettifiche influenzano in maniera diversificata il Capitale a seconda del metodo di calcolo adottato:

- nel metodo Standard viene riconosciuta la differenza tra rettifiche di valore generiche (Generic Provisions), che possono concorrere al Capitale (nel Tier 2 fino all' $1,25 \%$ delle esposizioni ponderate per il rischio) e quelle poste a fronte di rischi specifici (Specific Provisions), dunque non computabili nel capitale (e dedotte dal valore regolamentare dell'EAD).

- nei metodi basati sui Rating Interni ("IRB") rileva la differenza tra due tipi di perdite su crediti con particolare riferimento agli eventi che possono manifestarsi nei 12 mesi successivi alla redazione del bilancio: la perdita attesa e la perdita inattesa. La prima è vista come una componente del business ed è, perciò gestita tramite il pricing dei prodotti e le svalutazioni, la seconda invece viene coperta dal Capitale, che svolge dunque una funzione di copertura dei picchi in eccesso rispetto alle perdite attese (fino ad una predefinita soglia di tolleranza). Pertanto, le rettifiche contabili vengono confrontate con la perdita attesa regolamentare: qualora le prime siano inferiori alle seconde si ha una carenza (shortfall) che richiede di essere coperta con Capitale CET 1; nel caso contrario si rileva un eccesso (excess) di accantonamenti che viene riconosciuto come capitale di Tier 2 (fino ad un massimo dello $0.6 \%$ degli attivi ponderati per il rischio). 
L'introduzione dell'IFRS 9 modificherà l'interazione tra capitale e le riserve, dal momento che non bisognerà più attendere l'evidenza di impairment (incurred loss) per effettuare gli accantonamenti, ma saranno già contabilizzate le perdite attese (expected loss) su tutti gli strumenti finanziari in un'ottica forward looking. Inoltre, per i crediti che mostrano un significativo deterioramento dall'origination (stage 2) sarà richiesta la contabilizzazione delle perdite attese lungo l'intera vita residua dello strumento. Queste due novità non incluse nel concetto di "perdita attesa" regolamentare ad un anno (forward looking e lifetime expected loss), comporteranno un rischio di "double counting" nella quantificazione del capitale, in quanto già incluse nella componente di "perdita inattesa". Pertanto, in assenza di modifiche all'attuale regolamentazione, ci si attende una variazione negativa degli indici patrimoniale, pur senza che sia intervenuta alcuna modifica nel rischio dei portafogli. E' dunque importante che le regole prudenziali siano ricalibrate prima dell'entrata in vigore dell'IFRS 9 stabilita l'1/1/2018.

Date queste premesse, il Comitato di Basilea ha recentemente pubblicato per discussione una serie di proposte al quadro regolamentare relativo al metodo Standard, fornendo le seguenti alternative:

- conservare l'attuale trattamento regolamentare delle rettifiche contabili, inclusa la distinzione tra rettifiche generiche (GP) e specifiche (SP);

- introdurre una nuova definizione di GP e SP, cui si accompagni un trattamento obbligatorio a fini patrimoniali;

- rivisitare il trattamento regolamentare delle riserve rimuovendo la distinzione tra GP e SP introducendo la stima di una perdita attesa sulla base di coefficienti stabiliti dal regolatore con conseguente quantificazione di una shortfall/excess simile al metodo avanzato.

In merito alle proposte di cui sopra, nel paper si è dato spazio a simulazioni di impatto attraverso esemplificazioni per valutare le implicazioni che ciascuna potrebbe portare al sistema bancario e in particolare ai Gruppi che segnalano con metodo Standard. In sintesi, si può affermare che non sono osservabili in funzione dei diversi scenari significative differenze di impatto sul CET1 ratio, che subirà sempre e comunque un aggravio dalla prima applicazione del nuovo principio contabile. Si può notare, al contrario, una significativa volatilità del total capital ratio a seguito della computazione o meno delle Generic Provisions nell'ambito del Tier 2.

Con riferimento ai metodi avanzati, il Comitato non sembra propenso ad effettuare revisioni al framework prudenziale. Tuttavia, in questo caso e come già evidenziato, si rileva che l'introduzione dei modelli IFRS 9 comporta nelle perdite contabili alcuni effetti (la componente di perdita attesa oltre l'orizzonte di 1 anno, gli scenari point-in-time e forwardlooking) che determinerebbero un double counting rispetto alla perdita inattesa. Pertanto, si ritiene opportuno valutare una revisione anche in tale ambito, per il quale una possibile soluzione potrebbe essere l'introduzione di un trattamento simmetrico di excess/shortfall rispetto alla perdita attesa regolamentare. Il trattamento simmetrico consentirebbe, sostanzialmente, di neutralizzare l'impatto del IFRS 9 in termini di capitale. Esso risulterebbe giustificato dal fatto che, con l'introduzione degli IFRS 9, non vi è in realtà una variazione nel livello di rischio delle banche e quindi non si dovrebbe assistere ad alcun cambiamento nei livelli di capitalizzazione (peraltro già sensibilmente rafforzati negli ultimi anni). Peraltro, tale modifica mitigherebbe l'incoerenza tra l'orizzonte di rischio utilizzato per la perdita attesa regolamentare (un anno) e contabile (un anno o lifetime).

In conclusione, a seguito degli esercizi esemplificativi affrontati nel paper e sopra riepilogati sia con riferimento al metodo standard sia con riferimento ai metodi avanzati, il gruppo di lavoro si è spinto a quantificare una prima dimensione degli effetti sul capitale, nei diversi scenari, osservabile dall'applicazione degli IFRS 9. In particolare, a partire dalla documentazione pubblica al 31/12/2015 riportata nei report Pillar III insieme ad alcuni dati di dettaglio riportati nel recente Stress Test condotto dall'EBA, si è effettuata una quantificazione di massima degli impatti attesi dell'introduzione dell'IFRS 9 sui principali istituti bancari italiani. Di seguito sono riportati i passi operativi e le principali assunzioni adottate.

stima della perdita attesa ad un anno: moltiplicando EAD, PD e LGD medie riportati nelle tabelle di Pillar III.

- stima della perdita attesa lifetime: si proiettano le PD ad un anno da Pillar III su un orizzonte pluriennale utilizzando come "ancoraggio" le PD a medio-lungo termine stimate dall'agenzia di rating Moody's. La LGD è invece ipotizzata flat durante tutta la vita attesa.

- conversione in PIT(point-in-time): si applica alle PD regolamentari, per definizione TTC (through-the-cycle), un fattore di aggiustamento dato dal quoziente tra il default rate osservato nell'ultimo anno e il default rate complessivo osservato sul periodo di stima delle PD.

- staging allocation: operare tale allocazione sulla base della sola documentazione pubblica è rischioso e può portare a risultati fuorvianti, perché i dati sulla distribuzione per classe di rischiosità corrente dei crediti non sono sufficienti, visto che è necessario il confronto con la rischiosità all'origination degli strumenti finanziari (cd. "approccio relativo"). Si preferisce quindi affidarsi ad un'ipotesi arbitraria ma trasparente, assumendo che il $20 \%$ dei crediti si collochi in stage 2. 
Sulla base di tali ipotesi si osserva un incremento stimato delle rettifiche su crediti performing compreso tra il $30 \%$ e il 40\%. Si tratta di risultati soggetti a un consistente margine d'errore (considerata tra l'altro l'impossibilità di approssimare in maniera più puntuale gli esiti dello staging). Tuttavia, l'esercizio può dare un'idea di massima della dimensione degli impatti attesi.

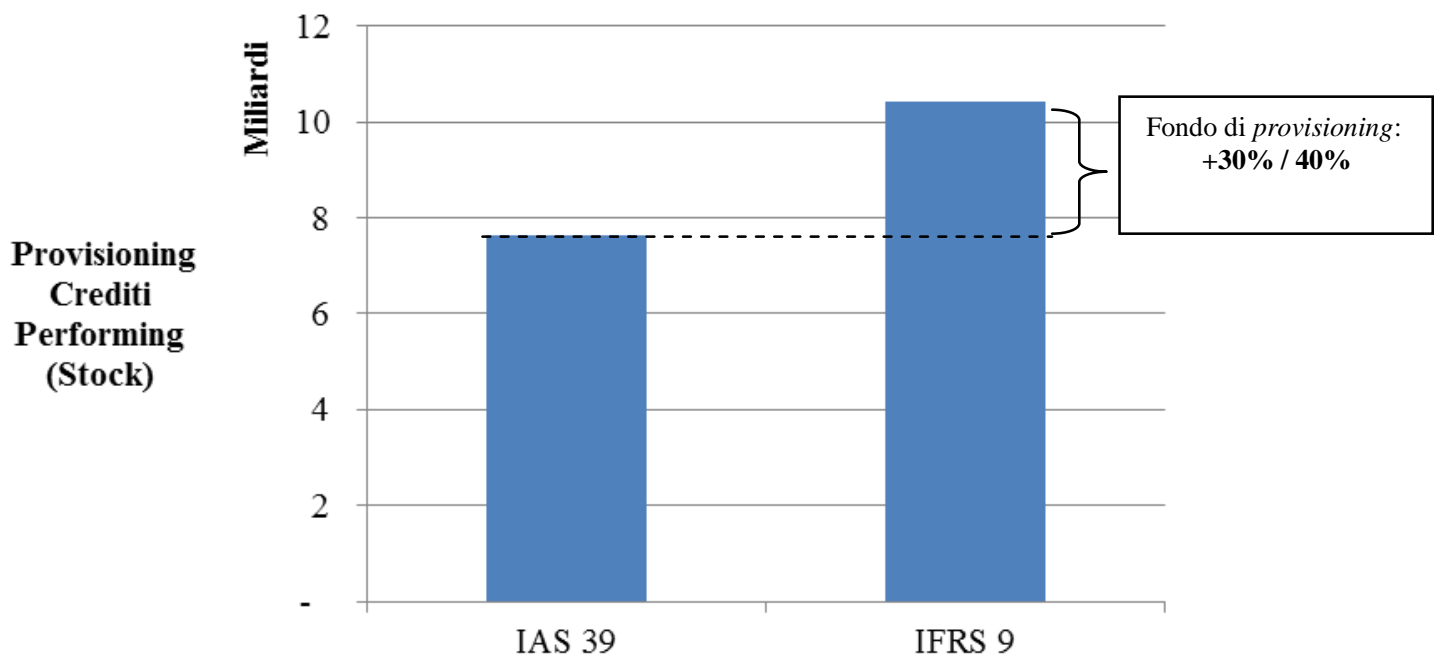

Figura 2 - Impatti attesi degli IFRS 9 sul livello delle Provisioning per i crediti performing

Si è quindi simulato l'impatto di tali maggiori accantonamenti sui ratio patrimoniali degli istituti, nei diversi scenari evolutivi della normativa prudenziale ipotizzati.

\begin{tabular}{|l|c|c|c|}
\hline & \multirow{2}{*}{$\begin{array}{c}\text { Conservare } \\
\text { l'attuale } \\
\text { trattamento }\end{array}$} & \multicolumn{2}{|c|}{ Nuova definizione di GP e SP } \\
\cline { 3 - 4 } & $-0.13 \%$ & $1^{\circ}$ variante & $2^{\circ}$ variante \\
\hline$\Delta \%$ Risk Weighted Asset & $-0.12 \%$ & $+0.33 \%$ & $+0.27 \%$ \\
\hline$\Delta \%$ CET 1 Ratio & $-0.12 \%$ & $-0.14 \%$ & $-0.14 \%$ \\
\hline$\Delta \%$ Total Capital Ratio & $+0.30 \%$ & $+0.24 \%$ \\
\hline
\end{tabular}

Tabella 1 - Portafogli Standard - Impatti attesi sugli indici patrimoniali

\begin{tabular}{|l|c|c|c|c|}
\hline & \multirow{2}{*}{$\begin{array}{c}\text { Conservare } \\
\text { l'attuale } \\
\text { trattamento }\end{array}$} & \multicolumn{2}{|c|}{ Trattamento simmetrico } & \multirow{2}{*}{$\begin{array}{c}\text { Trattamento } \\
\text { asimmetrico }\end{array}$} \\
\cline { 3 - 4 } & $0.00 \%$ & $0.00 \%$ & $0.00 \%$ & $-0.76 \%$ \\
\hline$\Delta \%$ Risk Weighted Asset & $-0.20 \%$ & $0.00 \%$ & $-0.20 \%$ & $-0.16 \%$ \\
\hline$\Delta \%$ CET 1 Ratio & $-0.01 \%$ & $0.00 \%$ & $0.00 \%$ & $-0.15 \%$ \\
\hline$\Delta \%$ Total Capital Ratio & & & su Tier 2 & \\
\hline
\end{tabular}

Tabella 2 - Portafogli AIRB - Impatti attesi sugli indici patrimoniali

Per le banche che adottano il metodo Standard, complessivamente potrebbe essere atteso un impatto sul CET1 ratio di 12-20bps (considerando altresì lo scenario di adozione di una EL regolamentare) e sul Total Capital ratio di 0-24bps. Con riferimento alle banche che adottano il metodo IRB, secondo l'attuale framework regolamentare, è atteso un effetto sul CET1 ratio intorno ai 20bp, mentre non sono attesi significativi impatti sul Total Capital ratio. Gli altri scenari mostrano effetti migliori sul CET1 ratio, ma anche potenzialmente peggiori sul Total Capital ratio. Come già evidenziato, si tratta di risultati soggetti a un consistente margine d'errore (considerata tra l'altro l'impossibilità di approssimare in maniera più puntuale gli esiti dello staging). Tuttavia, l'esercizio può dare un'idea di massima della dimensione degli impatti attesi. 
I principali impatti sui processi bancari derivanti dall'applicazione dell'IFRS 9

Come si è visto nel corso del documento, la conseguenza più scontata e diretta dell'IFRS 9 riguarda l'incremento delle rettifiche su crediti e in generale della complessità dei processi di provisioning con le conseguenze osservate sul capitale regolamentare. Tuttavia, gli impatti del nuovo principio riguardano potenzialmente molte fasi della gestione di un'istituzione finanziaria, sia con riferimento alla pianificazione delle politiche creditizie e della composizione del portafoglio ottimale, sia nelle attività di monitoraggio del credito.

Con riferimento ai processi direzionali:

- pianificazione e budget: sarà necessario garantire coerenza tra le ipotesi alla base delle stime del piano/budget e quelle utilizzate per stimare gli accantonamenti ai sensi del nuovo principio.

- performance measurement: la valutazione consistente delle performance corrette per il rischio può risultare più complessa, data la maggiore articolazione della nuova modellistica. Sarà, dunque, necessario attrezzarsi per individuare uno o più "key performance indicators" (KPI) coerenti con la nuova definizione di ECL (Expected Credit Losses).

- politiche creditizie: l'introduzione di una logica forward looking nelle valutazioni contabili impone una riflessione sulle attuali politiche di selezione della clientela. Anche nella credit risk mitigation sarà importante definire policy che premino l'acquisizione di collateral a minore volatilità e sensitività rispetto al ciclo economico, per contenere gli impatti in termini di ECL.

- Altri processi: comunicazione e disclosure verso gli stakeholder, reportistica interna e verso le Autorità di Vigilanza.

Anche i processi di business ne risulteranno coinvolti. Per esempio, nell'ambito del processo di concessione sarà necessario ridefinire le tipologie di prodotti e forme tecniche "vendibili" (il c.d. "catalogo" prodotti), tenendo conto anche della possibile classificazione in stage 2. Il rischio di passaggio a un ECL lifetime potrebbe infatti rendere estremamente onerosa l'erogazione di prodotti a medio lungo termine. Anche in tema di modelli di pricing risk adjusted andranno adeguati alle nuove logiche di valutazione contabile.

Per quanto concerne l'attività di monitoraggio del credito essa si arricchirà di nuovi criteri e paradigmi di intervento; verrà orientata anche a garantire la minimizzazione degli impatti sui livelli di accantonamento attraverso l'identificazione di una serie di azioni preventive e di mitigazione volte a ridurre la probabilità di passaggio in stage $2 \mathrm{e}$ gli impatti di tali passaggi.

Infine, a riguardo della capital adequacy andranno fatte riflessioni sulle interrelazioni tra rettifiche contabili e quantificazione del capitale, e delle sue prospettiche evoluzioni sopra riportate. Anche il processo ICAAP avrà degli impatti, in cui andrà mantenuta la coerenza tra la misurazione del capitale e la misurazione degli accantonamenti.

Nicola Pasturi e Luca Giuseppe Paulicelli 
Bibliografia

- AIFIRM (2017), Il principio contabile IFRS 9 in banca: la prospettiva del Risk Manager

I coordinatori della Commissione Aifirm:

Andrea Resti e Luca Giuseppe Paulicelli

I coodinatori dei sottogruppi di lavoro:

Paolo Costantino Cicorella, Giovanna Compagnoni, Silvio Cuneo, Elisabetta Fregoni, Nicola Pasturi, Roberta Rossi, Natale Schettini e Giuliana Tioli

e tutti gli oltre 80 iscritti alla Commissione che hanno portato il loro contributo.

Pubblicazioni citate nel lavoro:

- $\quad$ BCBS. (2015). Guidance on credit risk and accounting for expected credit losses.

- Board of Governors of the Federal Reserve System Office of the Comptroller of the Currency, Supervisory Guidance on Model Risk Management, SR Letter 11-7, 4 Aprile 2011

- $\quad$ Cerved - Experian (luglio 2016) "IFRS 9 - Simulazioni d'impatto sugli accantonamenti e possibili interventi sui processi del credito"

- $\quad$ CRIF (2016). Impairment dei crediti nell'IFRS 9: sfide e soluzioni, CRIF Group, Bologna

- EBA, Consultation Paper - Draft Guidelines on credit institutions' credit risk management practices and accounting for expected credit losses, EBA/CP/2016/10, 26 Luglio 2016

- $\quad$ EBA, EBA FINAL draft Regulatory Technical Standards on prudent valuation under Article 105(14) of Regulation (EU) No 575/2013 (Capital Requirements Regulation - CRR), EBA/RTS/2014/06/rev1, 23 gennaio 2015

- EBA, Guidelines on common procedures and methodologies for the supervisory review and evaluation process (SREP), EBA/GL/2014/13, 19 dicembre 2014

- $\quad$ Ernst\&Young “IFRS 9 - impairment. Il nuovo Standard contabile - prima analisi degli impatti sul business bancario".

- Global Public Policy Committee (GPPC), The implementation of IFRS 9 impairment requirements by banks, 17 giugno 2016

- $\quad$ GPPC (2016), The implementation of IFRS 9 impairment requirements by banks, 17 June 2016

- Hazewinkel, Michiel, ed. (2001), "Weibull distribution" Encyclopedia of Mathematics, Springer

- $\quad$ IASB (2005). IFRS 7 - Financial Instruments: disclosures, Agosto 2005 (e successivi aggiornamenti)

- $\quad$ IASB (2014). IFRS 9 Financial Instruments.

- IASB (2015). IFRS Staff Paper ITG (Transition Resource Group for Impairment of Financial Instruments), Incorporation of forward-looking scenarios,

- $\quad$ Koenker, R. (2005). Quantile Regression. Cambridge University Press.

- $\quad$ Mapping Between Both Rating Types, New York University Salomon Center and Wiley Periodicals, New York

- $\quad$ Matthews, B. (1975). Comparison of the predicted and observed secondary structure of T4 phage lysozyme. Biochim Biophys Acta., 20;405(2):442-51.

- $\quad$ Norris, J. R. (1997), Markov chains, Cambridge University Press, New York

- Olivier de Bandt, Nicolas Dumontaux, Vincent Martin, Denys Médée (2013), Stress-testing banks' corporate credit portfolio, Banque de France - Autorité de Contrôle Prudentiel, Direction des Etudes

- Pardoux E. (2008), Markov processes and applications : algorithms, networks, genome, and finance, Chichester : Wiley/Dunod, Wiley series in probability and statistics

- Sheskin, D. (2003). Handbook of Parametric and Nonparametric Statistical Procedures. CRC Press.

- Siarka P., (2011) Vintage analysis as a basic tool for monitoring credit risk, No7(14), Mathematical Economics

- $\quad$ Stanghellini, E. (2009). Introduzione ai metodi statistici per il credit scoring. Springer Science \& Business Media.

- $\quad$ Topp R., Perl R. (2010), Through-the-Cycle versus Point-in-Time Ratings and Implications of the Mapping Between Both Rating Types, New York University Salomon Center and Wiley Periodicals, New York 
Ulteriori risorse disponibili in rete:

- $\quad$ AIFIRM, Prudent Valuation Guidelines and Sound Practices, Marzo 2016

- Deloitte, IFRS 9 - Financial Instruments - http://www.iasplus.com/en/standards/ifrs/ifrs9\#link14

- $\quad$ https://www.aba.com/Tools/Offers/Documents/Vintage\%20Analysis\%20Basics\%20Whitepaper\%20\%20Sageworks\%20ABA.pdf

- $\quad$ KPMG: Financial instruments - Introducing IFRS 9 https://home.kpmg.com/xx/en/home/insights/2014/07/ith-2014-13.html

- The basics of vintage analysis https://www.aba.com/Tools/Offers/Documents/Vintage\%20Analysis\%20Basics\%20Whitepaper\%20\%20Sageworks\%20ABA.pdf

- Weibull analysis - http://www.mathpages.com/home/kmath122/kmath122.htm 


\section{Abstract}

The paper presents a set of measures to support and link up national initiatives for the digitisation of industry and related services across all sectors and to boost investment through strategic partnerships and networks. The European Commission proposes concrete measures to speed up the development of common standards in priority areas, such as $5 \mathrm{G}$ communication networks or cybersecurity. As companies integrate digital technologies into more business processes, they will accrue benefits like agility, competitiveness, reaction time and reach. They will also expose themselves to greater security threats related to digital business innovation, and they will need a digital risk officer (DRO). The paper is divided in four parts: introducing to the digital transformation, the DRO role, the measurement of the sectoral risk and the measurement of the enterprise risk.

In particular our paper describes the DRO role and how the digital business innovation requires a new look at enterprise risk, then presents a case study on the Industry ICT, with an approach of technical analysis of the risk management. For the sector analysis, the credit risk assessment is based on rating system and a set of financial and economic ratio will be analyzed for industrial sectors to assess the probability of default using Altman method, based on multiple discriminant statistical methodology. For the enterprise analysis, the paper presents an application of the VaR (Value at Risk) market model to the analysis of market risk management related to an investment ICT portfolio. Our goal is to provide an accurate assessment of the risk management of equity investments before and after Brexit.

\section{Introduzione: il contesto della digital transformation}

La Commissione europea ha presentato recentemente un pacchetto di misure per sostenere e collegare le iniziative nazionali per la digitalizzazione dell'industria e dei servizi connessi in tutti i settori e per stimolare gli investimenti attraverso reti e partenariati strategici. Ha proposto inoltre misure concrete per accelerare lo sviluppo di norme tecniche comuni nei settori prioritari, quali le reti di comunicazione $5 \mathrm{G}$ o la cybersecurity e modernizzare i servizi pubblici.

Il Commissario responsabile per l'economia e le società digitali ha dichiarato che l'Europa possiede una base industriale molto competitiva ed è leader in settori importanti a livello mondiale ma potrà conservare un ruolo guida solo se l'industria verrà digitalizzata in modo rapido ed efficace. È necessario uno sforzo comune a livello europeo per attirare gli investimenti necessari per la crescita dell'economia digitale.

Nell'attuale rivoluzione digitale è necessario sviluppare e realizzare ad un livello adeguato il potenziale di tecnologie come il cloud computing, la data science (data-driven) e l'internet delle cose, affinché le imprese che mirano a espandersi nel mercato unico possano contare su tre fattori chiave: un'immediata innovazione tecnologica, nuove norme e nuovi servizi pubblici digitali che rispondano alle esigenze odierne. In particolare:

\section{a)Digitalizzazione dell'industria}

Molti comparti economici stanno adottando rapidamente le tecnologie e i nuovi processi digitali ma l'industria europea deve sfruttare ancora meglio le opportunità digitali in tutti i settori e indipendentemente dalle dimensioni dell'impresa per essere competitiva a livello mondiale. I settori tradizionali (come l'edilizia, il settore agro-alimentare, il comparto tessile e la siderurgia) e le PMI sono particolarmente indietro per quanto riguarda la trasformazione digitale. Recenti studi ${ }^{3}$ indicano che la digitalizzazione dei prodotti e dei servizi incrementerà le entrate annuali delle imprese di oltre 110 miliardi di euro in Europa nei prossimi cinque anni.

Diversi Stati membri dell'UE hanno già varato strategie per sostenere la digitalizzazione dell'industria ma è necessario un approccio globale a livello europeo per evitare la frammentazione dei mercati e beneficiare delle evoluzioni del digitale come l'internet delle cose.

In questo senso la Commissione si propone di:

- contribuire a coordinare le iniziative nazionali e regionali in materia di digitalizzazione dell'industria mantenendo un dialogo continuo a livello europeo con tutte le parti coinvolte. Un quadro di governance sarà istituito dagli Stati membri e dall'industria;

- concentrare gli investimenti nei partenariati pubblico-privato dell'UE e incoraggiare fortemente il ricorso alle opportunità offerte dal piano di investimenti dell'UE e dai Fondi strutturali e di investimento europei;

- investire 500 milioni di euro in una rete paneuropea di poli di innovazione digitale (centri di eccellenza nelle tecnologie) in cui le imprese potranno ricevere consigli e sperimentare le innovazioni digitali;

\footnotetext{
Ministero Sviluppo Economico, Direzione generale per la politica industriale, la competitività e le Pmi, fabrizio.carapellotti@sviluppoeconomico.gov.it

${ }^{2}$ Fondo pensione agenti professionisti di assicurazione, romina.potenziani@gmail.com

${ }^{3}$ PwC, "Opportunities and Challenges of the industrial internet" (2015) e Boston Consulting Group, "The future of productivity and growth in manufacturing industries" (2015).
} 
- definire progetti pilota su larga scala per potenziare l'internet delle cose, i processi produttivi avanzati e le tecnologie in ambiti quali città intelligenti, domotica, automobili connesse o sanità mobile;

- $\quad$ adottare una normativa adeguata alle esigenze future che faciliti il libero flusso dei dati e chiarisca i diritti di proprietà dei dati generati da sensori e dispositivi intelligenti. La Commissione intende inoltre riesaminare le norme sulla sicurezza e l'affidabilità dei sistemi autonomi;

- $\quad$ presentare un'agenda europea per le competenze che contribuisca a dotare i cittadini delle competenze necessarie per i posti di lavoro nell'era digitale.

L'iniziativa europea per il cloud computing si inserisce nel contesto di questo pacchetto e aiuterà l'Europa ad assumere una posizione di primo piano nell'economia basata sui dati. Nel complesso i piani presentati dovrebbero mobilitare più di 50 miliardi di euro di investimenti pubblici e privati a sostegno della digitalizzazione dell'industria.

b)Norme tecniche prioritarie per incoraggiare l'innovazione digitale

Nel mercato unico digitale miliardi di dispositivi connessi, compresi i telefoni, i computer e i sensori, dovrebbero poter comunicare in modo sicuro e senza problemi, indipendentemente dal fabbricante, dai dettagli tecnici o dal paese d'origine. Per questo hanno bisogno di una lingua comune basata su norme tecniche, pertanto la Commissione propone misure concrete per accelerare il processo di definizione di tali norme:

- concentrarsi su cinque settori prioritari nel momento in cui si chiede all'industria e agli organismi di normazione di stabilire le norme: 5G, cloud computing, internet delle cose, tecnologie dei dati e cybersecurity;

- cofinanziamento di prove e sperimentazioni di tecnologie per accelerare l'elaborazione delle norme tecniche anche attraverso partenariati pubblico-privato, per garantire la definizione tempestiva di norme tecniche allo scopo di stimolare l'innovazione e la crescita delle imprese.

- l'approccio più rapido e più mirato consentirà inoltre di accelerare lo sviluppo e l'adozione di tecnologie quali le reti elettriche intelligenti, i servizi di sanità mobile, i veicoli connessi e altri settori. L'UE prevede di sostenere la partecipazione di esperti europei nelle decisioni di normazione internazionali affinché le idee europee contribuiscano a soluzioni globali.

c) Servizi pubblici digitali

I cittadini e le imprese non traggono ancora pienamente vantaggio dai servizi pubblici digitali che dovrebbero essere facilmente disponibili in tutta l'UE. Il piano d'azione per l'eGovernment permetterà di modernizzare i servizi pubblici digitali, contribuendo a migliorare lo stile di vita e facilitando il lavoro e gli investimenti. La Commissione propone 20 misure da varare entro la fine del 2017 , in particolare:

- $\quad$ istituire uno sportello digitale unico che permetta agli utenti di ottenere tutte le informazioni, l'assistenza e i servizi di risoluzione dei problemi necessari a operare in modo efficiente a livello transfrontaliero;

- connettere tra di loro tutti i registri delle imprese e i registri fallimentari e collegarli al portale della giustizia elettronica che diverrà uno sportello unico;

- definire un progetto pilota con le amministrazioni per applicare il principio di "una tantum" per le imprese a livello transfrontaliero; ciò significa che le imprese dovranno fornire la documentazione alle autorità pubbliche in un solo paese dell'Unione, anche se opereranno in altri Stati membri dell'UE;

- $\quad$ aiutare gli Stati membri a sviluppare servizi di sanità elettronica transfrontalieri come le prescrizioni e le cartelle cliniche elettroniche;

- $\quad$ accelerare la transizione verso gli appalti elettronici, le firme elettroniche e l'attuazione del principio "una tantum" negli appalti pubblici.

In questo nuovo contesto digitale gli Stati membri dell'Unione Europea, che fino ad oggi sono stati liberi di affrontare la questione della sicurezza informatica e della protezione dei dati come meglio ritenevano, devono attivare pratiche di risk management per rilevare proattivamente violazioni imminenti o comportamenti rischiosi da parte degli utenti. Infatti la vastità di dati condivisi attraverso i confini interni dell'UE implica che l'impatto del crimine informatico e di altre forme di violazioni dei dati è aumentato considerevolmente, rendendo necessaria una direttiva sulla cybersecurity.

Una procedura per segnalare le violazioni dovrebbe essere definita e verificata in modo indipendente. Deve esserci consapevolezza che la quantità di dati inviati da e verso dispositivi mobili sta esplodendo, il che sottolinea la necessità di un'efficace sicurezza mobile. Le regole e gli standard di conformità già esistono e in effetti molti fornitori stanno già offrendo da tempo prodotti che consentano di conformarsi alla direttiva sulla cybersecurity.

E' necessario spingere le imprese a capire che il cyber risk non è solo un rischio IT ma una minaccia globale per l'azienda e che proprio alla luce di ciò il risk manager può rivestire un ruolo primario nella quantificazione dei rischi. Oggi le sfide maggiori per le imprese riguardano il rischio reputazionale, la sicurezza della supply chain e l'attuazione di strategie sostenibili di mitigazione del rischio, ma moltissimi business hanno difficoltà a raggiungere un livello base di protezione finanziaria contro il rischio cyber proprio perché manca un'analisi efficace, mancano dati certi e strategie di mitigazione funzionali.

Il contributo, dopo aver introdotto la figura del digital risk officer, che ricopre un ruolo determinante in un contesto sempre più digitale dove è fondamentale per l'impresa un'attenta valutazione dei rischi, si propone di misurare il rischio di credito e il rating per i settori industriali, e di analizzare il rischio di mercato per le società che operano nel comparto ICT, individuando sia il profilo di rischio che le perdite attese di portafoglio delle società quotate. Si ricorre all'applicazione di tecniche di risk management che forniscono, con il contributo di specifiche metodologie di misurazione del rischio, strumenti di gestione utili per una scelta d'investimento efficiente, attraverso le seguenti analisi: 
1) Settoriale: prendendo in esame i principali indicatori economico finanziari per settore viene applicato un metodo di credit scoring (metodo di Altman) per elaborare un indice di rischio di insolvenza (score) che consente di stimare la probabilità di default (PD) di ogni comparto settoriale.

2) Aziendale: Sulla base dei titoli azionari delle società esaminate è stata effettuata una analisi di portafoglio nell'ottica di studiare e misurare i rischi di mercato connessi ad un investimento azionario di imprese del settore ICT.

Il lavoro dunque è diviso in quattro parti: l'introduzione sul contesto della digital transformation, il ruolo del digital risk officer, la misura del rischio settoriale, la misura del rischio aziendale con la valutazione del portafoglio di società del comparto ICT.

\section{2 - Il ruolo del digital risk officer}

Le aziende sono impegnate a integrare le tecnologie digitali in altri processi di business, al fine di ottenere maggiori benefici in termini di agilità, competitività e rapidità. Potranno dunque esporsi a maggiori minacce alla sicurezza legate all'innovazione digitale del proprio business. Queste minacce però richiederanno una risposta più sofisticata e un portafoglio notevolmente ampliato al di là di rischi tradizionali e ruoli di sicurezza utilizzati fino ad oggi. Forse la più grande sfida è quella di cambiare la mentalità comune che la tecnologia digitale sia un problema tecnico gestito solo dal settore IT.

Dunque l'innovazione digitale richiede una nuova figura professionale, il Digital Risk Officer (DRO). Tale figura, che dovrà far fronte principalmente alla gestione dei processi IT, dei nuovi modelli di business e delle varie tipologie di rischi (tra cui quelli legati alla sicurezza informatica), risponderà ad una figura superiore al di fuori dell'IT, come può essere il Chief Risk Officer, oppure il Chief Digital Office, i quali poi tratteranno il rischio coinvolgendo le business unit digitali che lavorano con il reparto legale, con il reparto che si occupa della privacy, con il digital marketing e con il settore vendite digitali. Le diverse figure che si occupano di rischi di sicurezza aziendale evolveranno verso questa nuova figura di Digital Risk Officer.

I DRO in questo contesto hanno bisogno di creare rapporti di lavoro di qualità superiore in modo che possano contribuire ai nuovi modelli di business.

Parti del modello di business digitale in espansione di cui si avvarranno le organizzazioni sono il cloud, il mobile, l'Internet of Things (IoT), le tecnologie operative (OT), telefonia, audio e video. In tal modo l'azienda andrà incontro ad un rischio complessivo più elevato che minaccia l'integrità dei processi di business e di erogazione dei servizi. Quindi il nuovo modello di business digitale richiede un approccio integrato nel quale operare in collaborazione con altre unità, quali lo sviluppo scientifico, il marketing, l'ufficio legale e protezione dati, consente di massimizzare le sinergie e una gestione coordinata delle varie tipologie di rischi.

Il DRO avrà il compito di eseguire le valutazioni dei rischi della tecnologia in tutta l'azienda digitale per meglio supportare le decisioni nei processi di business e rilevarne l'impatto, prestazioni e strategia. Ad esempio una valutazione potrebbe includere il sistema che raccoglie informazioni personalmente identificabili dalla tecnologia wearable, i dati utilizzati nel marketing e operazioni di vendita, questioni di privacy e la monetizzazione dei dati come una fonte di reddito.

Al fine di fornire un'efficace sistema di sicurezza al DRO verrà richiesto un mix tra senso degli affari e conoscenze tecniche sufficienti per valutare il rischio in una impresa digitale integrata, e per affrontare questo rischio in modo da non ostacolare il modello di business digitale.

I DRO dovranno conoscere tecnologie e processi di business digitali tra cui:

- Cloud, mobile, social e big data

- Tecnologie per la sicurezza fisica

- Internet delle cose (Internet of Things)

- Tecnologie operative (Operational Technologies)

- Sicurezza Machine-to-machine (M2M)

- La gestione della sicurezza fisica

- Software embedded e sistema di sicurezza

- Identità e gestione degli accessi (IAM)

- Recupero associato con la consegna di business digitale

Allo stesso tempo dovrà avere anche una vasta conoscenza dei processi di business, aspetti normativi e valutazione del rischio tra cui:

- gestione della continuità operativa

- privacy

- requisiti legali e normativi

- valutazioni del rischio su modelli di business digitali end to end

- conoscenza di finanza e contabilità

- digital marketing e customer intimacy

- problemi di sicurezza della supply chain

I DRO devono essere dotati di eccellenti doti di comunicazione, forte conoscenza del business e la capacità di tradurre le questioni molto tecniche in fattori di decisione connessi alle imprese. Dovranno gestire il rischio ad un livello esecutivo attraverso unità di business digitali lavorando direttamente con i colleghi che si occupano di materie giuridiche, di privacy, di digital marketing, di vendite digitali e di operazioni digitali. 
Un DRO in grado di progettare ed eseguire un coerente approccio integrato offrirà maggiori garanzie di rischio per i processi di business rispetto all'approccio frammentario attualmente in atto in molte aziende.

Influenzeranno in questo modo la governance, la supervisione e il processo decisionale relativi al business digitale. Le strutture commerciali dovranno essere riprogettate con la priorità in materia di sicurezza e la necessità di un esecutivo esperto nella valutazione del rischio, del monitoraggio, dell'analisi e del controllo.

Il DRO ispirerà quindi l'equilibrio tra la necessità di proteggere l'organizzazione e la necessità di gestire l'attività.

\section{3 - La misura del rischio settoriale}

L'evoluzione dell'approccio di risk management ha visto un allargamento sempre più ampio e complesso delle varie tipologie di rischio cui l'impresa è esposta, e di conseguenza delle relative competenze e professionalità necessarie oggi per assolvere al ruolo di digital risk officer. Un'evoluzione che ha portato verso competenze più gestionali, che da un lato richiedono una maggiore conoscenza del business e dei suoi processi chiave così come dei mercati finanziari, e dall'altro una solida base quantitativa a supporto delle necessarie modellizzazioni per la misurazione e la gestione del rischio.

La mappatura dei rischi dell'impresa è un'attività oggi estremamente articolata che passa attraverso un'attenta valutazione dei processi di business, del posizionamento sui mercati, del modello organizzativo e del rapporto con il sistema finanziario.

L'esposizione al rischio generalmente viene ricondotta a tre principali categorie: rischi strategici, rischi operativi e rischi finanziari. All'interno della categoria dei rischi finanziari assumono un ruolo importante il rischio di mercato, che vedremo nel prossimo paragrafo, e il rischio di credito, inteso come deterioramento delle posizioni creditorie, derivanti sia da attività commerciali che da attività finanziarie, con un conseguente incremento della probabilità di insolvenza.

L'obiettivo dell'analisi settoriale è proprio quello di valutare il merito creditizio delle imprese di un determinato settore, elaborando una misura del rating e della relativa probabilità di default (PD). In particolare è stato utilizzato un modello statistico di scoring, noto come Z-score di Altman, che si basa su indicatori economico finanziari provenienti dai bilanci di 2060 società censite da Mediobanca, per la misurazione del rischio di credito delle imprese classificate in termini settoriali.

Sulla base di tale modello è infatti possibile prevedere la probabilità di fallimento o il merito creditizio di un'impresa.

La funzione di Altman è utilizzata sempre più frequentemente per analizzare sinteticamente l'equilibrio finanziario di un'azienda o di un settore. Si basa sull'attribuzione di punteggi che consentono di esprimere un giudizio qualitativo di posizionamento dell'azienda rispetto a cinque criteri indicatori della solvibilità aziendale: la flessibilità, la capacità di autofinanziamento, l'analisi della redditività e il livello di indipendenza da terzi.

La formulazione più recente è quella del modello EM-score e la funzione utilizzata per il calcolo del valore dell'EMscore è la seguente:

$\mathrm{EM}=3.25+6.56 \mathrm{X} 1+3.26 \mathrm{X} 2+6.72 \mathrm{X} 3+1.05 \mathrm{X} 4$

dove:

- $\mathrm{X} 1=($ Capitale Circolante Netto $) /($ Capitale Investito $)$

è un indice di flessibilità aziendale;

- $\quad \mathrm{X} 2=($ Utili Non Distribuiti) / (Capitale Investito)

è un indice di autofinanziamento;

- $\mathrm{X} 3=$ (Risultato Operativo $) /($ Capitale Investito $)$

è un indice di redditività;

- $\quad$ X4 = (Valore di Mercato) / (Passività totali)

è un indice di indipendenza da terzi.

Al crescere del valore EM diminuisce la probabilità d'insolvenza. Sulla base del valore dell'EM-score è possibile costruire una classificazione del rating e stimare il corrispondente valore della probabilità di default (PD) degli ultimi 5 anni nel modo seguente:

Fig. 1 Formula per la stima della probability of default (PD)

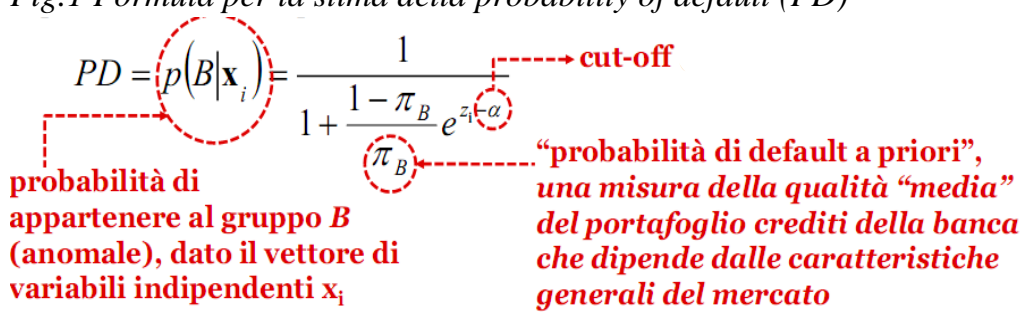


Tab.1 Classificazione del rating del valore EM-score

\begin{tabular}{ll}
\hline Classi Score EM & Rating \\
\hline EM $\geq 8.15$ & AAA \\
$7.60 \leq E M<8.15$ & AA+ \\
$7.30 \leq E M<7.60$ & AA \\
$7.00 \leq E M<7.30$ & AA- \\
$6.85 \leq E M<7.00$ & A+ \\
$6.65 \leq E M<6.85$ & A \\
$6.40 \leq E M<6.65$ & A- \\
$6.25 \leq E M<6.40$ & BBB+ \\
$5.85 \leq E M<6.25$ & BBB \\
$5.65 \leq E M<5.85$ & BBB- \\
$5.25 \leq E M<5.65$ & BB+ \\
$4.95 \leq E M<5.25$ & BB \\
$4.75 \leq E M<4.95$ & BB- \\
$4.50 \leq E M<4.75$ & B+ \\
$4.15 \leq E M<4.50$ & B \\
$3.75 \leq E M<4.15$ & B- \\
$3.20 \leq E M<3.75$ & CCC+ \\
$2.50 \leq E M<3.20$ & CCC \\
$1.75 \leq E M<2.50$ & CCC- \\
EM $<1.75(a l f a)$ & D \\
\hline$F O n t e: ~$
\end{tabular}

Fonte: In-Depht Data Corp.(over 750 U.S. Corporates with rated debt outstanding: 1995 data)

Di seguito viene riportato l'EM-score ottenuto per settore industriale.

Tab.2 Valore EM-score 2006-2015

\begin{tabular}{|c|c|c|c|c|c|c|c|c|c|c|c|}
\hline SETTORI & 2006 & 2007 & 2008 & 2009 & 2010 & 2011 & 2012 & 2013 & 2014 & 2015 & \begin{tabular}{|c|} 
media \\
2011-2015 \\
\end{tabular} \\
\hline SOCIETA' INDUSTRIALI & 4,83 & 4,60 & 4,59 & 4,60 & 4,68 & 4,47 & 4,52 & 4,53 & 4,59 & 4,72 & 4,57 \\
\hline Alimentare & 5,00 & 5,04 & 5,02 & 5,11 & 5,21 & 5,14 & 5,01 & 5,31 & 5,38 & 5,71 & 5,31 \\
\hline Tessile & 6,07 & 6,04 & 5,46 & 5,29 & 5,50 & 5,63 & 5,76 & 5,62 & 5,92 & 6,05 & 5,80 \\
\hline Abbigliamento & 5,59 & 5,41 & 5,32 & 5,90 & 6,07 & 5,96 & 6,21 & 6,03 & 6,24 & 6,50 & 6,19 \\
\hline Pelli e cuoio & 6,15 & 5,74 & 5,75 & 5,55 & 5,80 & 6,33 & 6,56 & 6,60 & 6,09 & 6,55 & 6,43 \\
\hline Legno e mobili & 5,09 & 5,14 & 5,09 & 4,72 & 5,02 & 4,81 & 4,72 & 4,57. & 4,69 & 5,10 & 4,78 \\
\hline Cartario & 4,05 & 4,22 & 4,28 & 4,60 & 4,36 & 4,35 & 4,21 & 4,06 & 4,18 & 4,27 & 4,21 \\
\hline Stampa - editoria & 5,29 & 4,85 & 4,69 & 4,25 & 4,44 & 4,34 & 3,24 & 3,56 & 3,47 & 3,28 & 3,58 \\
\hline Petrolio, derivati e altri combustibili & 5,81 & 5,35 & 5,14 & 4,97 & 4,99 & 4,88 & 5,20 & 5,27 & 5,20 & 5,43 & 5,20 \\
\hline Chimico & 5,31 & 5,23 & 4,92 & 4,99 & 5,24 & 5,14 & 5,06 & 5,29 & 5,25 & 5,75 & 5,30 \\
\hline Farmaceutico e cosmetico & 5,92 & 5,72 & 5,67 & 5,95 & 6,08 & 5,91 & 6,24 & 6,26 & 6,52 & 7,26 & 6,44 \\
\hline Gomma e cavi & 4,11 & 4,36 & 3,63 & 3,95 & 4,06 & 3,96 & 4,16 & 3,53 & 3,76 & 3,44 & 3,77 \\
\hline Vetro & 5,12 & 5,86 & 5,63 & 4,41 & 4,46 & 4,67 & 4,61 & 5,13 & 5,12 & 5,26 & 4,96 \\
\hline Prodotti per l'edilizia & 5,69 & 5,63 & 5,31 & 5,35 & 5,09 & 4,67 & 4,66 & 4,89 & 4,86 & 4,72 & 4,76 \\
\hline Metallurgico & 5,47 & 6,01 & 5,39 & 4,66 & 4,85 & 4,85 & 4,71 & 4,61 & 4,91 & 5,07 & 4,83 \\
\hline Meccanico & 5,01 & 5,05 & 4,95 & 4,78 & 4,97 & 4,89 & 4,71 & 4,65 & 4,91 & 4,99 & 4,83 \\
\hline Eettrodomestici appar. radio-tv & 4,33 & 4,36 & 4,55 & 4,51 & 4,32 & 4,11 & 3,94 & 4,22 & 3,87 & 4,00 & 4,03 \\
\hline Eettronico & 5,03 & 4,62 & 4,75 & 4,44 & 4,91 & 4,50 & 4,43. & 4,53 & 4,42 & 5,38 & 4,65 \\
\hline Mezzi di trasporto & 3,80 & 3,63 & 3,58 & 3,33 & 3,59 & 2,90 & 2,91 & 2,98 & 3,44 & 3,49 & 3,14 \\
\hline Imprese di costruzione & 4,85 & 4,75 & 4,77 & 4,67 & 4,68 & 4,51 & 4,65 & 4,39 & 4,45 & 4,42 & 4,49 \\
\hline Energia elettrica e gas & 4,44 & 3,75 & 4,17 & 4,53 & 4,54 & 4,16 & 4,37 . & 4,24 & 4,09 & 4,01 & 4,17 \\
\hline Telecomunicazioni & 4,12 & 3,86 & 3,29 & 3,70 & 3,99 & 3,49 & 3,44 & 3,18 & 3,28 & 3,29 & 3,34 \\
\hline Società diverse & 4,58 & 4,28 & 4,42 & 4,29 & 4,47 & 4,19 & 4,10 & 4,29 & 4,74 & 4,84 & 4,43 \\
\hline SOCIETA' TERZIARIE & 4,14 & 3,96 & 3,69 & 3,71 & 3,82 & 3,57 & 3,47 & 3,50 & 3,59 & 3,68 & 3,56 \\
\hline
\end{tabular}

Fonte: elaborazioni su dati Mediobanca

Una volta ottenuti i valori dell'EM score, vengono attribuiti i relativi giudizi qualitativi di rating sulla base della scala di classificazione del rating del valore EM (tab.1). Successivamente la probability of default (PD) di ogni settore è stata stimata utilizzando il valore medio degli ultimi 5 anni, 2011-2015, dell'EM-score che corrisponde al valore $\mathrm{z}$ nella formula in fig.1.

Dai dati elaborati nel graf.1 emerge che i settori con il più alto valore della PD sono quelli dei mezzi di trasporto, delle telecomunicazioni, delle società terziarie e della stampa-editoria. 


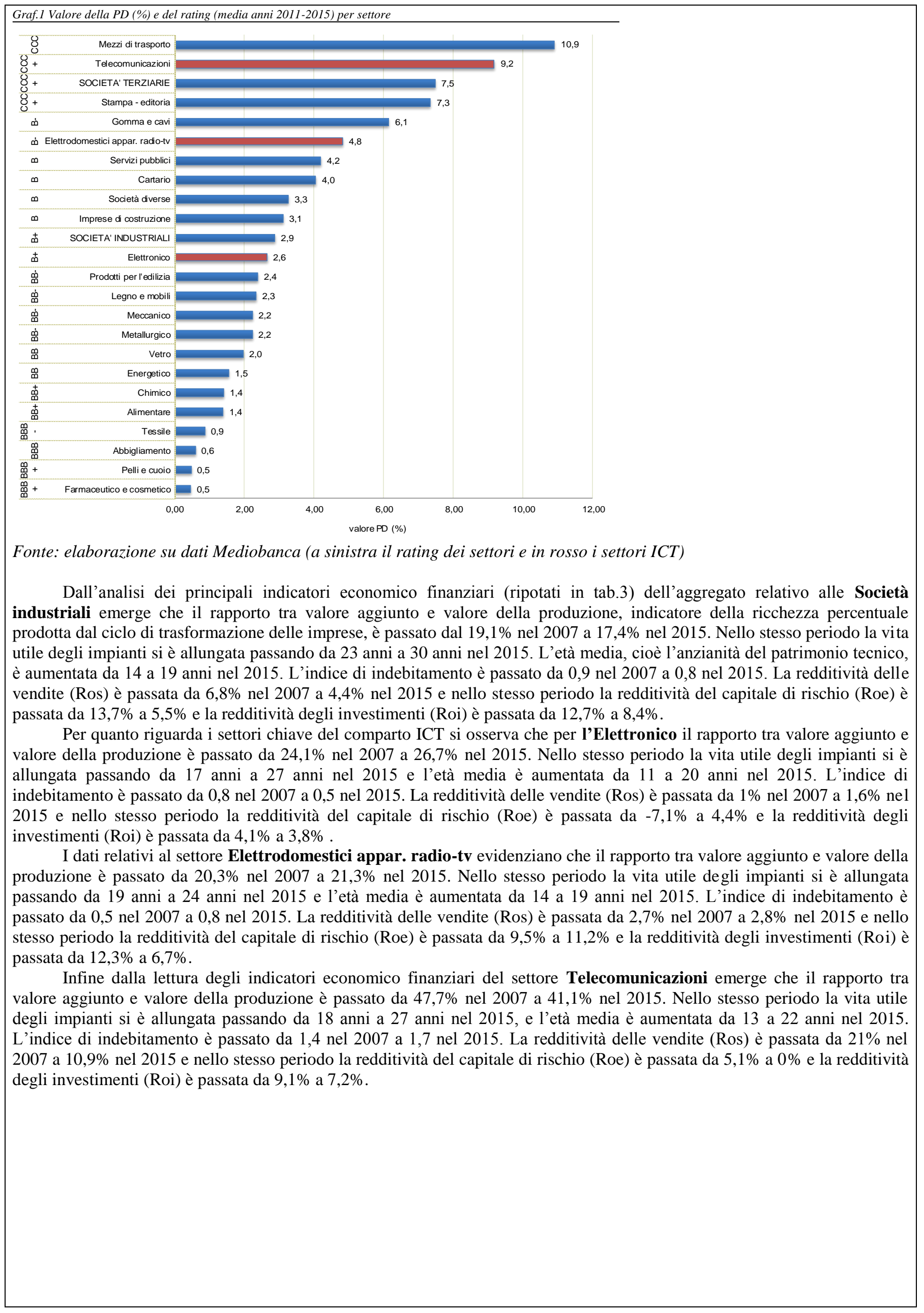




\begin{tabular}{|c|c|c|c|c|c|c|c|c|}
\hline \multirow{2}{*}{ INDICATORI } & \multicolumn{2}{|c|}{ Società industriali } & \multicolumn{2}{|c|}{ Elettronico } & \multicolumn{2}{|c|}{ Elettrodomestici } & \multicolumn{2}{|c|}{ Telecomunicazioni } \\
\hline & 2007 & 2015 & 2007 & 2015 & 2007 & 2015 & 2007 & 2015 \\
\hline QUOTA DIFATTURATO ALL'EXPORT, \% & 33,2 & 42,4 & 45,9 & 50,7 & 59,9 & 65,9 & 5,5 & 5,0 \\
\hline VALORE AGGIUNTO SU VALORE DELLA PRODUZIONE, \% & 19,1 & 17,4 & 24,1 & 26,7 & 20,3 & 21,3 & 47,7 & 41,1 \\
\hline VALORE AGGIUNTO SU FATTURATO NETTO, \% & 20,1 & 18,6 & 25,9 & 28,2 & 21,2 & 22,6 & 52,3 & 45,8 \\
\hline VALORE AGGIUNTO PRO-CAPTE (EURO) & 104.291 & 102.584 & 69.955 & 76.738 & 59.431 & 69.579 & 241.247 & 210.359 \\
\hline VITA UTILE DEGLI IMPANTI (ANNI) & 22,6 & 29,8 & 16,6 & 27,4 & 19,1 & 23,9 & 17,6 & 26,7 \\
\hline ETA' MEDIA DEGLI IMPANTI (ANNI) & 13,8 & 19,3 & 11,4 & 20,3 & 13,9 & 19,4 & 13,3 & 21,8 \\
\hline FLUSSO DI CASSA ENTRANTE (Migliaia euro) & 22.786 .021 & 21.048 .595 & 58.479 & 380.451 & 200.951 & 381.305 & -618.680 & 6.276 .175 \\
\hline FLUSSO DI CASSA USCENTE (Migliaia euro) & 2.340 .187 & 16.933.473 & -958.169 & 1.629 .197 & -10.098 & 93.331 & 3.358 .552 & 5.167 .776 \\
\hline FLUSSO DI CASSA NETTO (Mgliaia euro) & 20.445 .834 & 4.115 .122 & 1.016 .648 & -1.248 .746 & 211.049 & 287.974 & -3.977 .232 & 1.108 .399 \\
\hline FLUSSO DI CASSA NETTO/FATTURATO, \% & 4,1 & 0,8 & 8,2 & $-12,4$ & 2,8 & 4,6 & $-9,7$ & 3,6 \\
\hline MAGAZZINO, GG & 65 & 83 & 96 & 82 & 42 & 44 & 6. & 5 \\
\hline DILAZIONE A CLIENTI, GG & 76 & 78 & 118 & 92 & 78 & 79 & 94 & 88 \\
\hline DILAZIONE DA FORNITORI, GG & 76 & 84 & 92. & 90 & 82 & 95 & 155 & 131 \\
\hline INDICE DI EQUILIBRIO FINANZIARIO (LEVERAGE) & 1,1 & 1,2 & 1,5 & 1,6 & 1,1 & 1,0 & 1,0 & 0,9 \\
\hline INDICE DI INDEBTAMENTO TOTALE & 0,9 & 0,8 & 0,8 & 0,5 & 0,5 & 0,8 & 1,4 & 1,7 \\
\hline INDICE DIAUTONOMIA FINANZIARIA & 0,3 & 0,4 & 0,3 & 0,4 & 0,4 & 0,4 & 0,3 & 0,3 \\
\hline INDICE DI LIQUIDITA' SECONDARIA & 1,1 & 1,2 & 1,3 & 1,4 & 1,1 & 1,0 & 0,9 & 0,6 \\
\hline INDICE DI LIQUIDITA' PRIMARIA & 0,8 & 0,9 & 0,9 & 1,1 & 0,8 & 0,8 & 0,9 & 0,6 \\
\hline ROS\% (Return on sales) & 6,8 . & 4,4 & 1,0 & 1,6 & 2,7 & 2,8 & 21,0 & 10,9 \\
\hline ROE\% (Return on equity) & 13,7 & 5,5 & $-7,1$ & 4,4 & 9,5 & 11,2 & 5,1 & 0,0 \\
\hline ROl\% (Return on investment) & 12,7 & 8,4 & 4,1 & 3,8 & 12,3 & 6,7 & 9,1 & 7,2 \\
\hline
\end{tabular}

Fonte: elaborazione su dati Mediobanca

Graf.2 Andamento del valore EM-score e del rating per i principali settori del comparto ICT
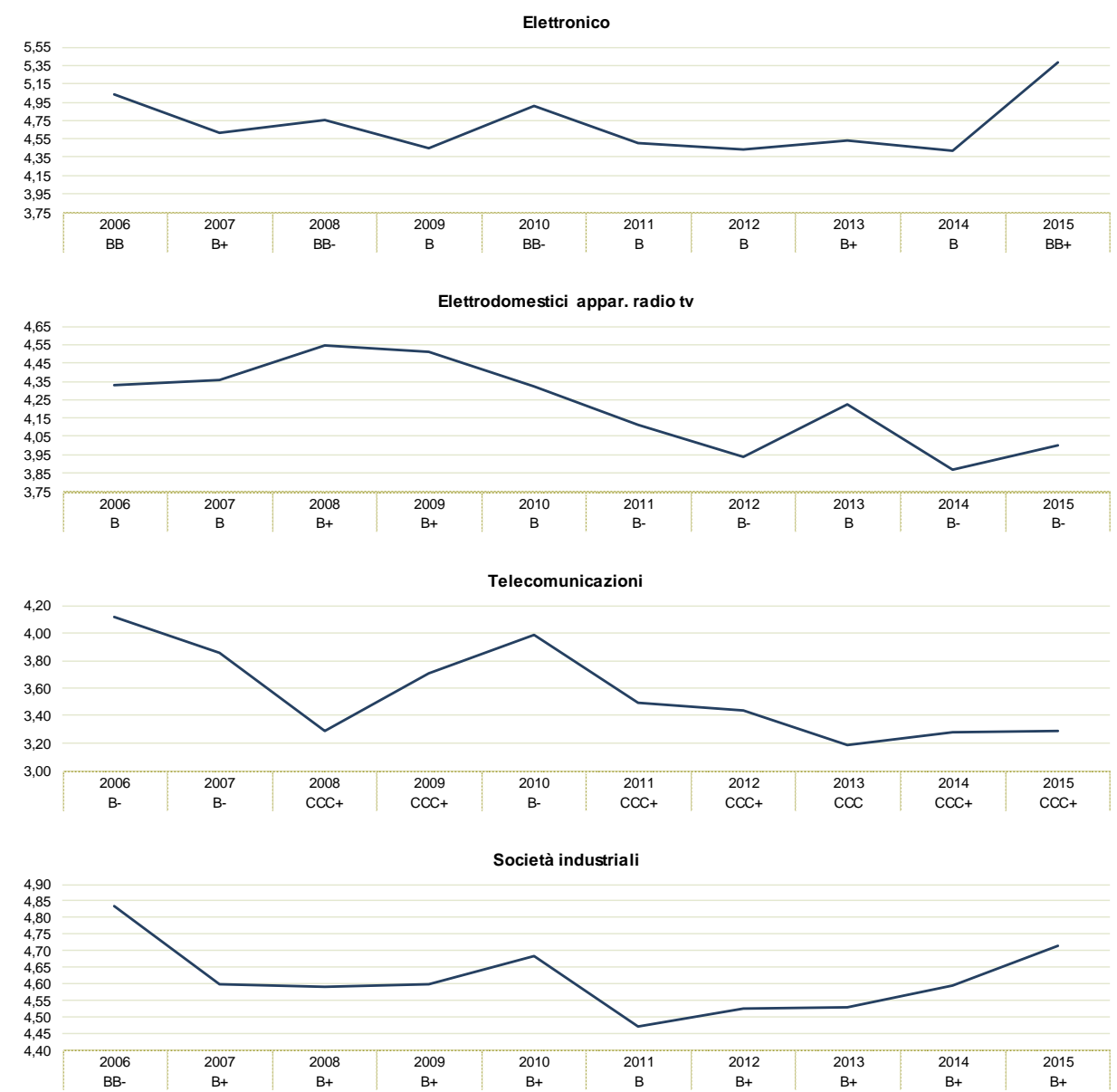

Fonte: elaborazione su dati Mediobanca

I modelli di scoring presentano comunque alcuni limiti che occorre tenere presente nel momento in cui essi vengono utilizzati per la stima della probabilità di insolvenza di un'impresa, in particolare:

1. Un primo problema importante riguarda il criterio di suddivisione del campione di stima fra imprese "sane" e imprese "insolventi", in realtà fra questi due estremi esistono diversi gradi di insolvenza. Quindi se si adottasse una definizione molto ampia, caratterizzata dall'inclusione fra le imprese insolventi anche di quelle che hanno registrato 
un semplice ritardo nel pagamento degli interessi, si otterrà un modello che classifica come insolventi un numero elevato di imprese;

2. Un secondo problema è legato al fatto che non vi è alcuna ragione economica per la quale il peso dei singoli indici economico-finanziari utilizzati come variabili esplicative dell'insolvenza debba rimanere immutata;

3. Un terzo problema è legato al fatto che i modelli di scoring trascurano numerosi fattori qualitativi che assumono invece particolare rilevanza nel determinare l'insolvenza di un'impresa;

4. Un quarto problema è legato al fatto che le imprese del campione di stima dovrebbero essere il più possibile omogenee in termini di settore produttivo di appartenenza.

\section{4 - La misura del rischio aziendale}

L'analisi aziendale si basa sui modelli VaR (Value at Risk) che assumono una rilevanza particolare in quanto consentono di valutare in maniera accurata il profilo di redditività e di rischio insito in ogni operazione di richiesta di finanziamento.

Questo metodo consente di effettuare un'analisi del rischio di mercato su un portafoglio di titoli azionari e di quantificare la perdita massima potenziale considerando oltre alla volatilità dei singoli titoli anche le correlazioni che esprimono il legame esistente tra le diverse posizioni detenute in portafoglio.

Il metodo VaR utilizzato viene definito asset-normal o Riskmetrics ${ }^{\mathrm{TM}}$ ed è utilizzato da molti operatori.

Tale modello è legato in particolare all'ipotesi di normalità dei rendimenti, la cui violazione può condurre all'elaborazione di stime poco attendibili del livello di rischio, ossia della massima perdita potenziale relativa all'insieme di attività finanziarie detenute in portafoglio.

Inoltre la composizione del portafoglio rimane costante nel periodo di tempo analizzato in modo che la volatilità del rendimento passato possa essere una buona previsione della volatilità futura.

In tale modello il Valore a Rischio di un portafoglio composto da $\mathrm{N}$ posizioni viene calcolato considerando sia le volatilità delle singole attività $\sigma$, che le correlazioni tra le diverse posizioni $\rho_{i j}$, oltre ai valori di mercato delle singole posizioni $\mathrm{VM}$, la costante $\mathrm{k}$ che individua l'intervallo di confidenza scelto e il periodo di detenzione $\mathrm{T}$, e viene formalizzato nel modo seguente:

$$
V a R_{P}=k \sqrt{\sum_{i=1}^{N} \sum_{j=1}^{N}\left(V M_{i} \cdot \sigma_{i}\right)\left(V M_{j} \cdot \sigma_{j}\right) \cdot \rho_{i j}}
$$

dove:

$V M_{i}, V M_{j}$ sono i valori di mercato delle posizioni i e $\mathrm{j}$

$\sigma_{i}, \sigma_{j} \quad$ sono le volatilità dei rendimenti delle posizioni i e j

$\rho_{i j} \quad$ è il coefficiente di correlazione tra le due posizioni

$\mathrm{T}=1 \quad$ periodo di detenzione è per semplicità pari a uno.

In generale se analizziamo un portafoglio con più di due posizioni risulta più agevole il calcolo del VaR asset-normal mediante l'algebra matriciale e quindi con la seguente formulazione:

$$
V a R=\sqrt{X^{\prime} \cdot \sum \cdot X} \cdot k \cdot \sqrt{T}
$$

dove:

$X$ è il vettore degli importi investiti nei singoli titoli e $X^{\prime}$ '̀ il corrispondente vettore trasposto

$\sum$ è la matrice Varianza-Covarianza dei titoli detenuti in portafoglio.

Il portafoglio costruito è rappresentativo delle aziende del settore ICT quotate su Borsa Italiana. Sono stati considerati i tre settori chiave identificati in base alla seguente classificazione ATECO: 


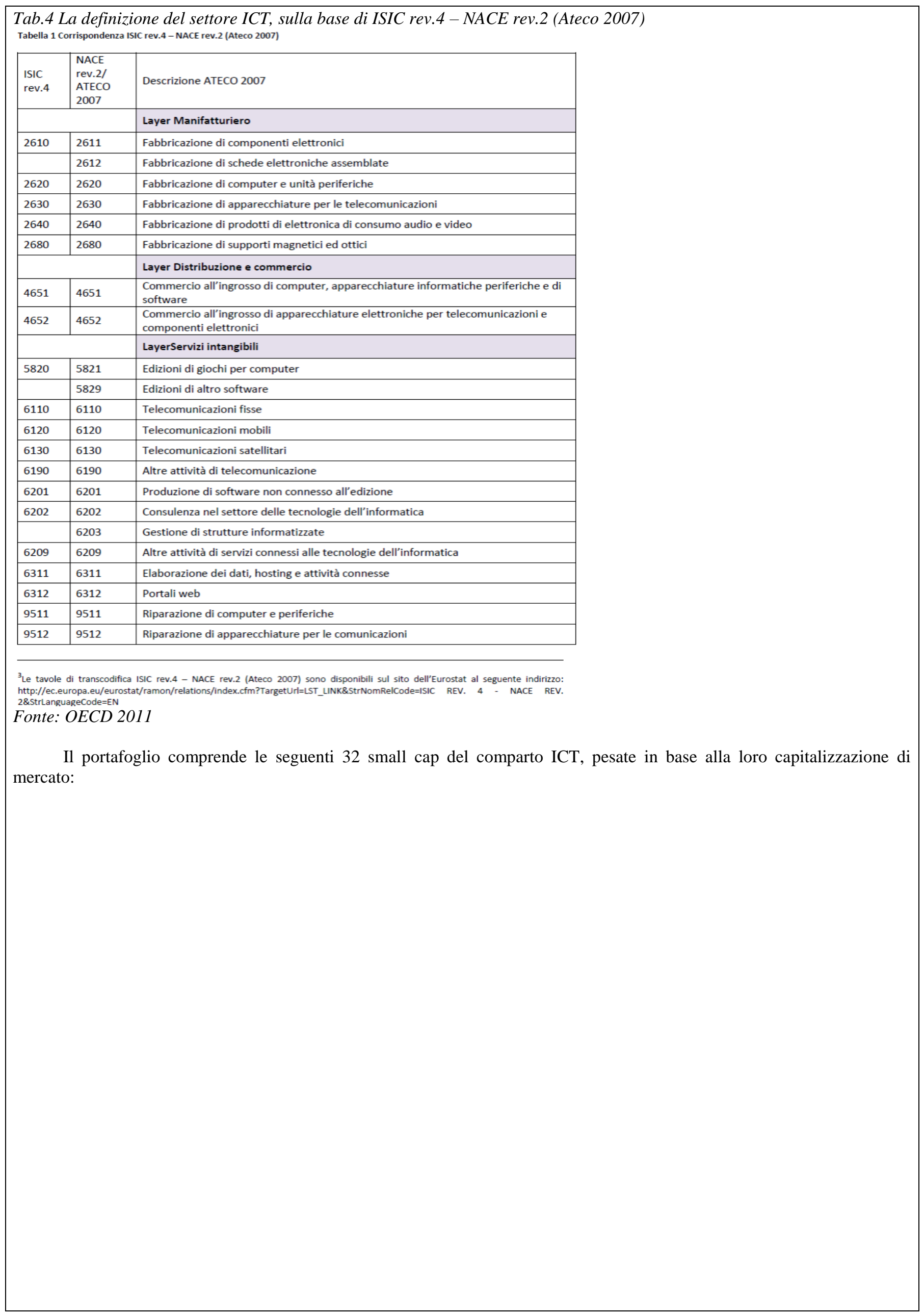


Tab.5 Le 32 small cap del comparto ICT

\begin{tabular}{|c|c|c|c|c|}
\hline Nome & Industry_sector & ICB_SUPERSECTOR & cod.nace & Market Cap \\
\hline INFRASTRUTTURE W & Communications & Telecommunications & 26.30 & 2.612 .400 .128 \\
\hline DATALOGIC SPA & Industrial & Industrial Goods \& Services & 62.0 & 1.073 .077 .568 \\
\hline REPLY SPA & Communications & Technology & 62.01 & 1.061 .549 .248 \\
\hline ESPRINET SPA & Consumer, Cyclical & Technology & 46.51 & 306.041 .344 \\
\hline SESA SPA & Technology & Technology & 62.0 & 229.288 .624 \\
\hline TISCALI SPA & Communications & Technology & 61.10 & 140.908 .624 \\
\hline DIGITAL BROS SPA & Technology & Personal \& Household Goods & 58.21 & 117.684 .384 \\
\hline RETELIT SPA & Communications & Telecommunications & 61.10 & 115.149 .728 \\
\hline TXT E-SOLUTIONS & Technology & Technology & 58.29 & 95.726 .000 \\
\hline B\&C SPEAKERS SPA & Industrial & Personal \& Household Goods & 26.40 & 78.320 .000 \\
\hline PTEECO SPA & Technology & Technology & 58.29 & 71.776 .976 \\
\hline $\mathrm{BE}$ & Communications & Technology & 62.02 & 69.337 .200 \\
\hline TBS GROUP SPA & Consumer, Non-cyclical & Health Care & 62.02 & 67.454 .736 \\
\hline NOEMALIFE SPA & Technology & Technology & 58.29 & 61.725 .532 \\
\hline EUROTECH SPA & Technology & Technology & 26.20 & 49.366 .940 \\
\hline IRCE SPA & Industrial & Industrial Goods \& Services & 26.1 & 48.380 .160 \\
\hline DADA SPA & Communications & Technology & 63.12 & 37.296 .636 \\
\hline VETRYA SPA & Communications & Technology & 61.30 & 34.663 .028 \\
\hline EXPRIVIA SPA & Technology & Technology & 58.29 & 33.594 .864 \\
\hline CAD IT SPA & Technology & Technology & 58.29 & 32.435 .760 \\
\hline MOBYT SPA & Communications & Telecommunications & 61.20 & 31.337 .162 \\
\hline TAS TECNOLOGIA & Technology & Technology & 58.29 & 28.527 .850 \\
\hline ACOTEL GROUP SPA & Technology & Telecommunications & 58.29 & 24.477 .900 \\
\hline MC LINK SPA & Communications & Technology & 61.10 & 16.504 .748 \\
\hline IT WAY SPA & Consumer, Cyclical & Technology & 46.51 & 11.383 .658 \\
\hline GO INTERNET SPA & Communications & Technology & 63.12 & 10.785 .168 \\
\hline TECH-VALUE SPA & Technology & Technology & 62.01 & 9.560 .376 \\
\hline OLIDATA SPA & Technology & Technology & 26.20 & 5.236 .000 \\
\hline SOFTEC SPA & Technology & Technology & 58.29 & 5.069 .709 \\
\hline CALEIDO GROUP SP & Communications & Media & 63.12 & 4.424 .690 \\
\hline PRIMI SUI MOTORI & Technology & Technology & 58.29 & 4.202 .238 \\
\hline VISIBILIA EDITOR & Consumer, Non-cyclical & Media & 63.12 & 1.670 .778 \\
\hline
\end{tabular}

Di seguito una rappresentazione grafica di prezzo, volatilità e rendimenti del portafoglio, studiandone l'andamento dal $1^{\circ}$ gennaio 2010 al 26 agosto 2016 : 
Fig.2 Trend di prezzo, volatilità, rendimenti e VaR del portafoglio

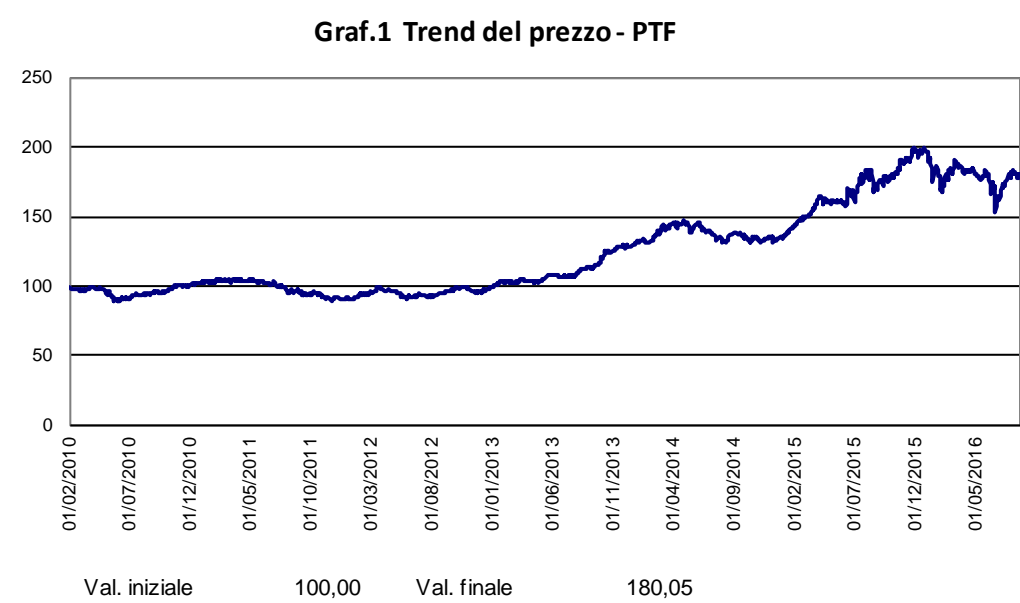

Graf.2 Volatilità 1 mese rolling

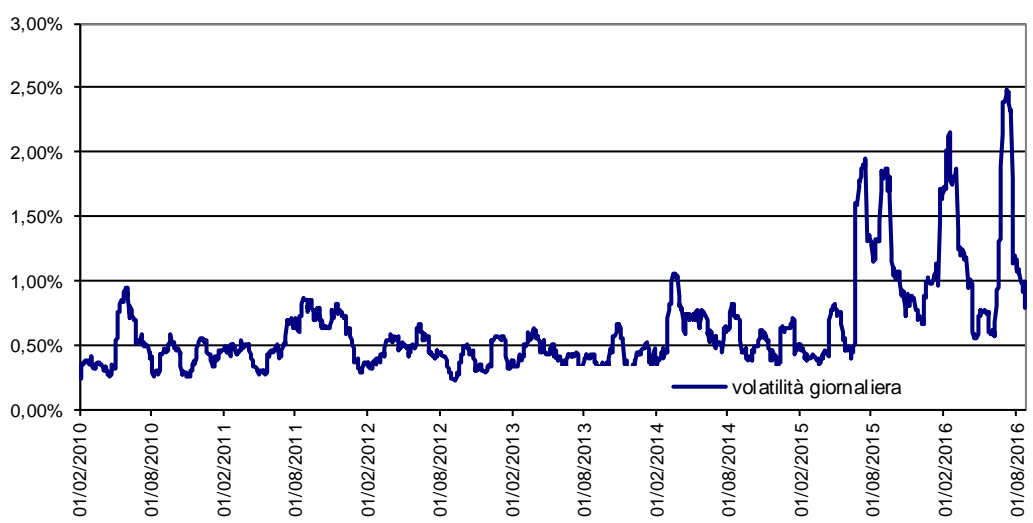

volatilità media giorn. $\quad 0,74 \%$

volatilità media annua $\quad 11,79 \%$

Graf.3 Rendimenti, VaR, Extreme tail return

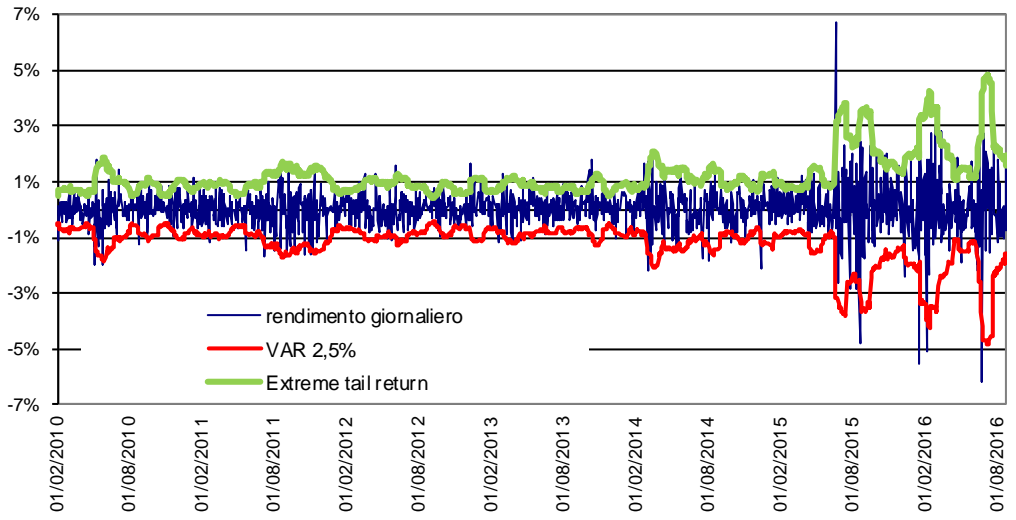

rendimento medio giorn. $\quad \begin{array}{lll}0,03 \% & \text { VaR medio giornaliero } & -1,46 \%\end{array}$

rendimento medio annuo $\quad 8,77 \% \quad$ VaR medio annuo $\quad 23,12 \%$

Fonte: elaborazioni su dati Yahoo finanza 
Tab.6 Rendimenti, volatilità e VaR delle 32 small cap

\begin{tabular}{|c|c|c|c|}
\hline Nome & $\begin{array}{c}\text { rendimento medio } \\
\text { annuo }\end{array}$ & $\begin{array}{c}\text { volatilità media } \\
\text { annua }\end{array}$ & VaR medio annuo \\
\hline REPLY SPA & $30 \%$ & $26 \%$ & $51 \%$ \\
\hline TXT E-SOLUTIONS & $24 \%$ & $38 \%$ & $74 \%$ \\
\hline DATALOGIC SPA & $22 \%$ & $28 \%$ & $54 \%$ \\
\hline DIGITAL BROS SPA & $19 \%$ & $50 \%$ & $98 \%$ \\
\hline B\&C SPEAKERS SPA & $16 \%$ & $29 \%$ & $57 \%$ \\
\hline RETEL T SPA & $7 \%$ & $42 \%$ & $83 \%$ \\
\hline SESA SPA & $7 \%$ & $18 \%$ & $35 \%$ \\
\hline NOEMALIFE SPA & $5 \%$ & $28 \%$ & $54 \%$ \\
\hline PTECO SPA & $3 \%$ & $8 \%$ & $16 \%$ \\
\hline INFRASTRUTTURE W & $2 \%$ & $11 \%$ & $22 \%$ \\
\hline IRCE SPA & $1 \%$ & $27 \%$ & $54 \%$ \\
\hline BE & $1 \%$ & $46 \%$ & $90 \%$ \\
\hline VETRYA SPA & $0 \%$ & $5 \%$ & $9 \%$ \\
\hline MOBYT SPA & $0 \%$ & $15 \%$ & $30 \%$ \\
\hline TECH-V ALUE SPA & $-6 \%$ & $17 \%$ & $33 \%$ \\
\hline GO INTERNET SPA & $-6 \%$ & $30 \%$ & $59 \%$ \\
\hline MC LINK SPA & $-6 \%$ & $26 \%$ & $50 \%$ \\
\hline ESPRINET SPA & $-6 \%$ & $41 \%$ & $81 \%$ \\
\hline CAD I SPA & $-7 \%$ & $31 \%$ & $61 \%$ \\
\hline TAS TECNOLOGIA & $-9 \%$ & $77 \%$ & $150 \%$ \\
\hline TBS GROUP SPA & $-9 \%$ & $38 \%$ & $74 \%$ \\
\hline EXPRIVIA SPA & $-10 \%$ & $33 \%$ & $65 \%$ \\
\hline EUROTECH SPA & $-11 \%$ & $36 \%$ & $70 \%$ \\
\hline T WAY SPA & $-13 \%$ & $42 \%$ & $83 \%$ \\
\hline DADA SPA & $-14 \%$ & $38 \%$ & $75 \%$ \\
\hline CALEIDO GROUP SP & $-14 \%$ & $15 \%$ & $30 \%$ \\
\hline OLIDATA SPA & $-18 \%$ & $53 \%$ & $104 \%$ \\
\hline TISCALI SPA & $-20 \%$ & $46 \%$ & $90 \%$ \\
\hline SOFTEC SPA & $-28 \%$ & $20 \%$ & $39 \%$ \\
\hline ACOTEL GROUP SPA & $-37 \%$ & $40 \%$ & $79 \%$ \\
\hline PRIMI SUI MOTORI & $-38 \%$ & $37 \%$ & $73 \%$ \\
\hline VISIBILIA EDITOR & $-64 \%$ & $57 \%$ & $112 \%$ \\
\hline
\end{tabular}

Fonte: elaborazioni su dati Yahoo finanza

Si nota la grande crescita del settore ICT negli ultimi anni, crescita accompagnata però da una forte volatilità data dall'instabilità dei mercati internazionali che ancora oggi continuano ad intaccare qualsiasi settore. Emergono i forti rendimenti di società come Reply, TXT e-Solution, Datalogic, Digital Bros e B\&C Speakers. descritto

I rendimenti si comportano come una distribuzione normale pertanto risulta attendibile l'analisi del VaR sopra

Il VaR giornaliero è stato tracciato stimando la volatilità giornaliera rolling ad un mese, in quanto avvalersi di periodi passati brevi consente di pervenire a previsioni migliori. Insieme ad esso è stato stimato anche il massimo guadagno potenziale.

Dalla fig.2 è facile notare come il portafoglio si è sempre mosso entro la stima di perdita massima potenziale, ad eccezione di alcuni periodi ad elevata incertezza e criticità a livello internazionale: agosto 2015 per la crisi Cinese, inizio anno 2016 con le gravi perdite subite da tutto il mercato Italia, e giugno 2016 per Brexit sullo scenario economico europeo.

Di contro però, il massimo guadagno potenziale non contiene tutti i picchi positivi registrati indice del fatto che il portafoglio con azioni del settore tecnologico è molto proficuo.

Con la presente analisi si è voluto evidenziare che nella gestione del rischio di credito delle imprese di un settore o del rischio di mercato di un portafoglio titoli la possibilità di incorrere rispettivamente in un peggioramento del merito creditizio o in forti perdite può essere stimata, e quindi prevista, anche in modo accurato, riuscendo quindi ad anticipare in tempi non sospetti le condizioni di crisi finanziaria di un'azienda o le sue potenzialità di crescita.

Molti analisti e studiosi del risk management attualmente stanno indirizzando i propri sforzi proprio nell'ottica predittiva, anche in considerazione del fatto che da un lato le banche stanno assumendo un comportamento sempre più prudenziale nella concessione del credito, in particolar modo verso alcune imprese che per dimensione e struttura possono manifestare maggiori difficoltà nel fornire garanzie a copertura delle esposizioni, e dall'altro i mercati finanziari si caratterizzano per una crescente volatilità e dunque rischiosità che richiede una più attenta analisi tecnica di un investimento azionario nelle imprese dell'era digitale. 
Riferimenti

- Altman E., (2000), Predicting financial distress of companies: revisiting the z-score and zeta models, Working Paper New York University

- EUROPEAN COMMISSION, (2016), Accelerating the digital transformation of European industry and enterprises.

- GARTNER, (2015), The Rise of the Digital Risk Officer, Gartner Security and Risk Management Summit

- Sda BocCONI, (2007), Definizione di un modello di scoring, Bocconi School of Management.

- Ufficio StUdi MediobancA, (2016), Indagine annuale sull'aggregato delle principali società italiane, Dati cumulativi di società italiane 


\title{
L'algoritmo Fuzzy C-Means clustering come tecnica automatica per l'individuazione di anomalie di mercato: un caso studio
}

di Pier Giuseppe Giribone ${ }^{1}$, Ottavio Caligaris ${ }^{2}$, Simone Fioribello ${ }^{3}$

\begin{abstract}
In literature there are several Data Mining methods being able to classify a different set of data in partitions designed to highlight similar characteristics. These algorithms are essential to carry out a segmentation of the population in order to distinguish common features among the elements belonging to the same class, on an automatic basis. Among all the clustering techniques, the ones relating to the field of soft-computing (such as Fuzzy Logic) are considered particularly suitable for application to the dynamic and unpredictable financial markets. The founding principles of Fuzzy Logic are well represented in the algorithm of Fuzzy C-Means: this technique allows, on an automatic basis, market data to be classified in real time, providing the operator not only an indication of the presence of potential anomalies (and therefore potential interesting trading opportunities), but also a percentage of reliability of such information. The study is divided into two parts: the first section deals with the principles of the Fuzzy C-Means clustering algorithm; in the second part a case study developed in Matlab environment has been shown, with regard to the identification of potential abnormalities in premium quotes of the Credit Default Swap (CDS) on a major Spanish bank.
\end{abstract}

Key Words:

Fuzzy C-Means (FCM) clustering, Fuzzy Logic (FL), soft-computing, Data Mining, Data Classification

\section{Introduzione}

In letteratura vengono presentate numerose metodologie di Data Mining finalizzate a classificare un insieme eterogeneo di dati in partizioni atte ad evidenziare caratteristiche omogenee. Tali algoritmi sono essenziali per effettuare una segmentazione della popolazione al fine di distinguere tratti comuni agli elementi appartenenti alla medesima classe, in via automatica ${ }^{4}$. Tra tutte le tecniche di clusterizzazione, si considerano particolarmente idonee ad essere applicate ai dinamici e difficilmente prevedibili mercati finanziari quelle afferenti al campo della soft-computing, (ad esempio la Fuzzy Logic $[3,6,11,13,14])$. I principi fondanti della logica sfumata sono ben rappresentati nell'algoritmo di partizione Fuzzy C-Means: tale tecnica permette, in via automatica, di classificare dati di mercato in tempo reale, fornendo all'operatore non solo l'indicazione sulla presenza di potenziali anomalie (e pertanto potenziali interessanti opportunità di trading), ma anche di associare una percentuale di affidabilità a tale informazione $[2,4]$.

Lo studio si suddivide in due parti: nella prima vengono trattati i principi di funzionamento dell'algoritmo Fuzzy C-Means clustering; nella seconda viene riportato un case-study sviluppato in ambiente Matlab, con riferimento all'individuazione di potenziali anomalie nelle quotazioni dei premi, espressi in basis points, dei Credit Default Swap (CDS) in applicazione ad un importante istituto creditizio spagnolo.

\section{Data Clustering: la tecnica Fuzzy C-Means}

Dato un insieme di dati, detto dataset, lo scopo del clustering è quello di identificare raggruppamenti naturali di questi punti, per cercare di ottenere una concisa rappresentazione del comportamento del sistema.

Proprio in quest'ottica, la tecnica di clustering che viene presentata e descritta in questo lavoro di ricerca è la cosiddetta Fuzzy C-Means (FCM): tale approccio metodologico, prendendo ispirazione direttamente dalla Fuzzy Logic ${ }^{5}$, è una tecnica di data clustering in cui, per ciascun punto del set di training si determina un certo grado di membership per ciascuno dei cluster che vengono individuati.

\footnotetext{
${ }^{1}$ BANCA CARIGE - Financial Engineering
}

${ }^{2}$ Professore ordinario di Analisi Matematica dell'Università di Genova - Dipartimento di Ingegneria Meccanica (DIME)

${ }^{3}$ Dottorando in Computer Science \& Systems Engineering - Università di Genova

${ }^{4}$ Per eventuali approfondimenti bibliografici inerenti gli algoritmi classici di clusterizzazione dati (dimostrazioni, funzionamento ed applicazioni) si rimanda in [16] nella sezione "Cluster Analysis"

${ }^{5}$ All'interno del presente articolo si sono volutamente omessi i principi di base della Fuzzy Logic poiché già trattati dagli autori nel loro precedente studio pubblicato sulla rivista dell'Associazione (Vol. 11, N.3, paragrafo "La modellizzazione di un sistema secondo i principi della logica sfumata" in "Implementazione della Fuzzy Logic per la gestione ottimale del portafoglio: la modellizzazione dell'avversione al rischio di un investitore attraverso tecniche di soft-computing". In termini teorici e generali, la metodologia vanta una amplissima letteratura specifica $[8,9,10,12,17]$. 
Sostanzialmente, dati $D$ punti, l'obiettivo è suddividerli in $N$ partizioni distinte; l'aspetto particolare e innovativo rispetto al clustering standard è che questo approccio consente di determinare quali sono i gradi di appartenenza (livelli di sfumatura) dell' $i$-esimo punto rispetto a ciascuna delle partizioni considerate.

L'algoritmo opera assegnando in maniera casuale un certo grado di membership $\left(\mu_{i j}\right)$ a ciascun punto $i$ rispetto ad ognuno dei $j$ cluster e ricavando di conseguenza una certa distribuzione spaziale iniziale dei centri di massa delle $N$ partizioni che si vogliono ricavare.

Attraverso un procedimento iterativo, la funzione muove dinamicamente i baricentri verso la localizzazione ottima, ovvero andando a minimizzare un funzionale di costo che rappresenta la somma delle distanze euclidee di ciascun punto da ciascun centro di massa, opportunamente pesate col corretto grado di membership.

Nell'ultima parte di questo paragrafo viene presentato l'algoritmo con cui opera la tecnica Fuzzy C-Means. Come già spiegato precedentemente, l'algoritmo si basa sulla minimizzazione del seguente funzionale di costo:

$$
J=\sum_{i=1}^{D} \sum_{j=1}^{N} \mu_{i j}\left\|x_{i}-c_{j}\right\|^{2}
$$

Dove:

$D=$ numero di punti del dataset;

$N$ = numero di cluster da dover individuare;

$x_{i}=i$-esimo punto del dataset;

$c_{j}=$ centro del $j$-esimo cluster;

$\mu_{i j}=$ grado di membership dell' $i$-esimo punto rispetto al $j$-esimo cluster.

A livello procedurale, si possono evidenziare 5 passi distinti che devono essere compiuti dall'euristica per raggiungere l'ottimo. [15]

1. Inizializzazione random di tutti i valori di membership $\mu_{i j}$

2. Calcolo dei centri di massa dei cluster mediante: $c_{j}=\frac{\sum_{i=1}^{D} \mu_{i j} x_{i}}{\sum_{i=1}^{D} \mu_{i j}}$

3. Aggiornamento di tutti i nuovi $\mu_{i j}$ mediante: $\mu_{i j}=\frac{1}{\sum_{k=1}^{N}\left(\frac{\left\|x_{i}-c_{j}\right\|}{\left\|x_{i}-c_{k}\right\|}\right)^{2}}$

4. Calcolo del valore del funzionale di costo $J$

5. Ripetizione dei passi 2-3-4 fino a che non si raggiunge un miglioramento pressoché nullo del funzionale di costo oppure si raggiunge il numero massimo di iterazioni imposto dallo sperimentatore.

\section{Applicazione della metodologia Fuzzy C-Means clustering all’individuazione di anomalie di mercato}

Il 29 gennaio 2016, alle ore 15.15, Bloomberg® forniva diverse quotazioni dei CDS senior su un'importante banca spagnola a $1,3,5,7$ e 10 anni.

I premi, espressi in basis points, forniti da diverse controparti, sono riportati nella tabella 1 e graficati in Figura 1. 


\begin{tabular}{|c|c|c|}
\hline ID points & Time & CDS [bps] \\
\hline 1 & 1 & 75,574 \\
\hline 2 & 1 & 76,95 \\
\hline 3 & 1 & 76,799 \\
\hline 4 & 1 & 80,7 \\
\hline 5 & 1 & 88,98 \\
\hline 6 & 1 & 79,903 \\
\hline 7 & 1 & 76,965 \\
\hline 8 & 1 & 85,459 \\
\hline 9 & 1 & 90,97 \\
\hline 10 & 1 & 86,831 \\
\hline 11 & 1 & 95,09 \\
\hline 12 & 1 & 102,02 \\
\hline 13 & 1 & 89,834 \\
\hline 14 & 1 & 87,567 \\
\hline 15 & 3 & 110,871 \\
\hline 16 & 3 & 101 \\
\hline 17 & 3 & 112,521 \\
\hline 18 & 3 & 106 \\
\hline 19 & 3 & 116,88 \\
\hline 20 & 3 & 116,871 \\
\hline 21 & 3 & 113,241 \\
\hline 22 & 3 & 118,659 \\
\hline 23 & 3 & 111 \\
\hline 24 & 3 & 120,309 \\
\hline 25 & 3 & 116 \\
\hline 26 & 3 & 124,46 \\
\hline 27 & 3 & 123,685 \\
\hline 28 & 3 & 121,09 \\
\hline 29 & 5 & 149,021 \\
\hline 30 & 5 & 149,75 \\
\hline 31 & 5 & 147,14 \\
\hline 32 & 5 & 150,267 \\
\hline 33 & 5 & 149,25 \\
\hline 34 & 5 & 155 \\
\hline 35 & 5 & 150,176 \\
\hline 36 & 5 & 149,216 \\
\hline 37 & 5 & 149 \\
\hline 38 & 5 & 156,425 \\
\hline 39 & 5 & 154,75 \\
\hline
\end{tabular}

\begin{tabular}{|c|c|c|}
\hline ID points & Time & CDS [bps] \\
\hline 39 & 5 & 154,75 \\
\hline 40 & 5 & 156,78 \\
\hline 41 & 5 & 157,738 \\
\hline 42 & 5 & 159,25 \\
\hline 43 & 5 & 160 \\
\hline 44 & 5 & 156,781 \\
\hline 45 & 5 & 157,239 \\
\hline 46 & 5 & 154,67 \\
\hline 47 & 7 & 171,841 \\
\hline 48 & 7 & 172 \\
\hline 49 & 7 & 175,742 \\
\hline 50 & 7 & 184,01 \\
\hline 51 & 7 & 184,03 \\
\hline 52 & 7 & 179 \\
\hline 53 & 7 & 176,532 \\
\hline 54 & 7 & 175,06 \\
\hline 55 & 7 & 181,457 \\
\hline 56 & 7 & 182 \\
\hline 57 & 7 & 186,112 \\
\hline 58 & 7 & 195,92 \\
\hline 59 & 7 & 195,94 \\
\hline 60 & 7 & 185,6 \\
\hline 61 & 7 & 186,794 \\
\hline 62 & 7 & 183,09 \\
\hline 63 & 10 & 188,15 \\
\hline 64 & 10 & 192 \\
\hline 65 & 10 & 192,857 \\
\hline 66 & 10 & 206,47 \\
\hline 67 & 10 & 206,52 \\
\hline 68 & 10 & 199,27 \\
\hline 69 & 10 & 193,643 \\
\hline 70 & 10 & 191,473 \\
\hline 71 & 10 & 197,793 \\
\hline 72 & 10 & 202 \\
\hline 73 & 10 & 203,322 \\
\hline 74 & 10 & 218,55 \\
\hline 75 & 10 & 205,94 \\
\hline 76 & 10 & 203,995 \\
\hline 77 & 10 & 199,535 \\
\hline
\end{tabular}

Tabella 1: Quotazioni contribuite, espresse in punti base, dei CDS senior analizzati 


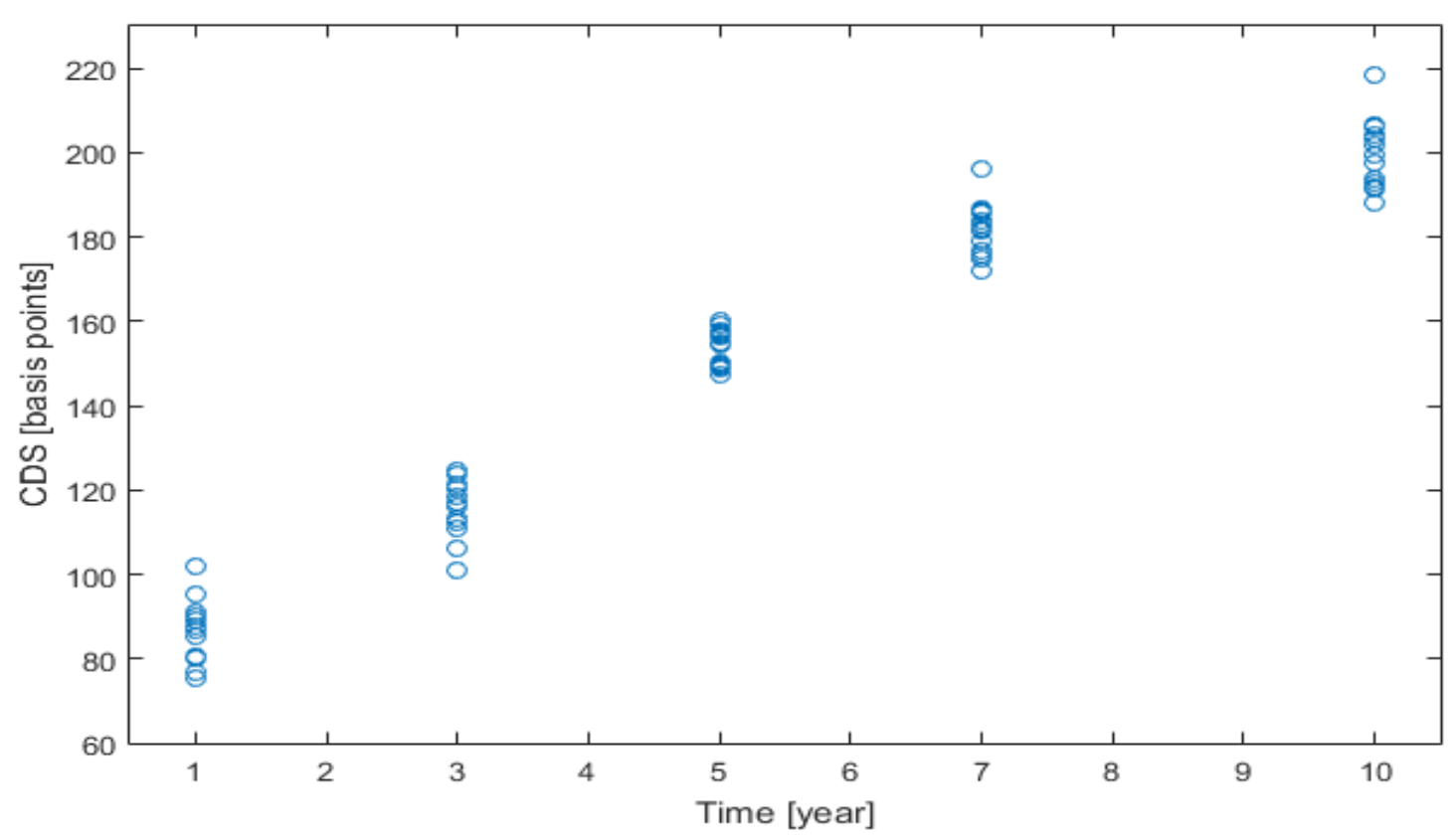

Figura 1: Rappresentazione grafica delle quotazioni dei CDS senior

Applicando l'algoritmo di clustering Fuzzy C-Means, descritto nel paragrafo precedente, sul dataset in analisi, si è in grado di evidenziare i centri di massa individuati dalla tecnica e riportati nella tabella 2.

La convergenza del metodo si ottiene dopo una dozzina di iterazioni della routine di minimizzazione principale (si confronti il grafico Iterazioni-Valori del funzionale di costo $J$ rappresentato nella Figura 2).

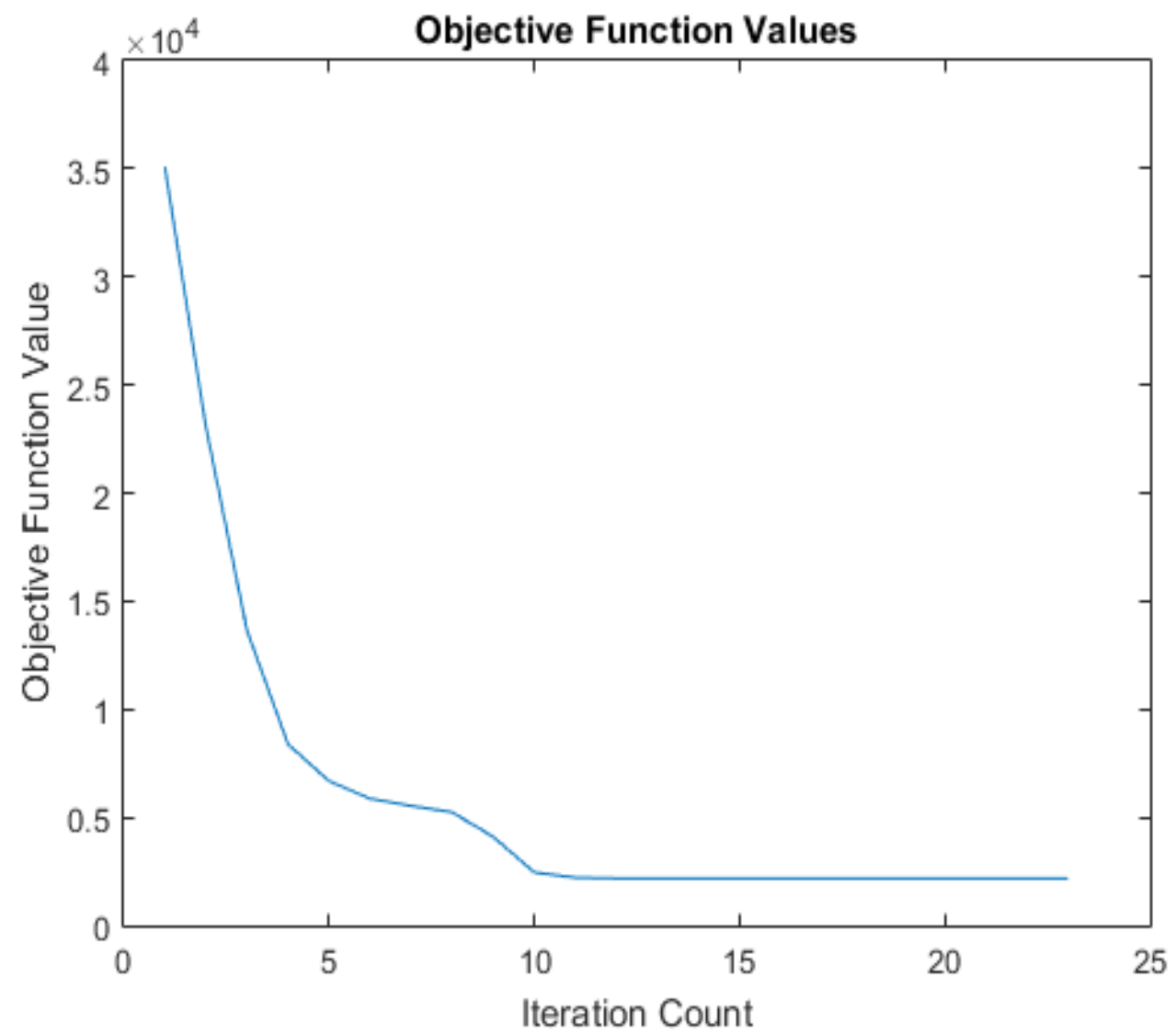

Figura 2: Grafico di convergenza dell'algoritmo di minimizzazione della funzione costo $J$ 


\begin{tabular}{|c|c|}
\hline $\mathbf{T}^{*}$ & CDS* \\
\hline 1,029815 & 84,0465 \\
\hline 2,93366 & 115,348 \\
\hline 5,011498 & 153,5278 \\
\hline 7,290601 & 181,9971 \\
\hline 9,64927 & 201,4692 \\
\hline
\end{tabular}

Tabella 2: Centri di massa individuati dalla tecnica Fuzzy C-Means

L'output della funzione permette di graficare i cluster individuati e fornisce la percentuale di appartenenza $\left(\mu_{i j}\right)$ dei punti a ciascun insieme. La Figura 3 mette in evidenza la partizione calcolata (cerchi evidenziati con colori d'appartenenza differenti) ed i relativi baricentri (le croci nere). I livelli di membership dei singoli CDS (rappresentati con l'identificativo numerico progressivo riportato nella Tabella 1) all'interno dei 5 cluster (identificati dalle ascisse dei loro centri di massa) sono riportati nella Tabella 3.

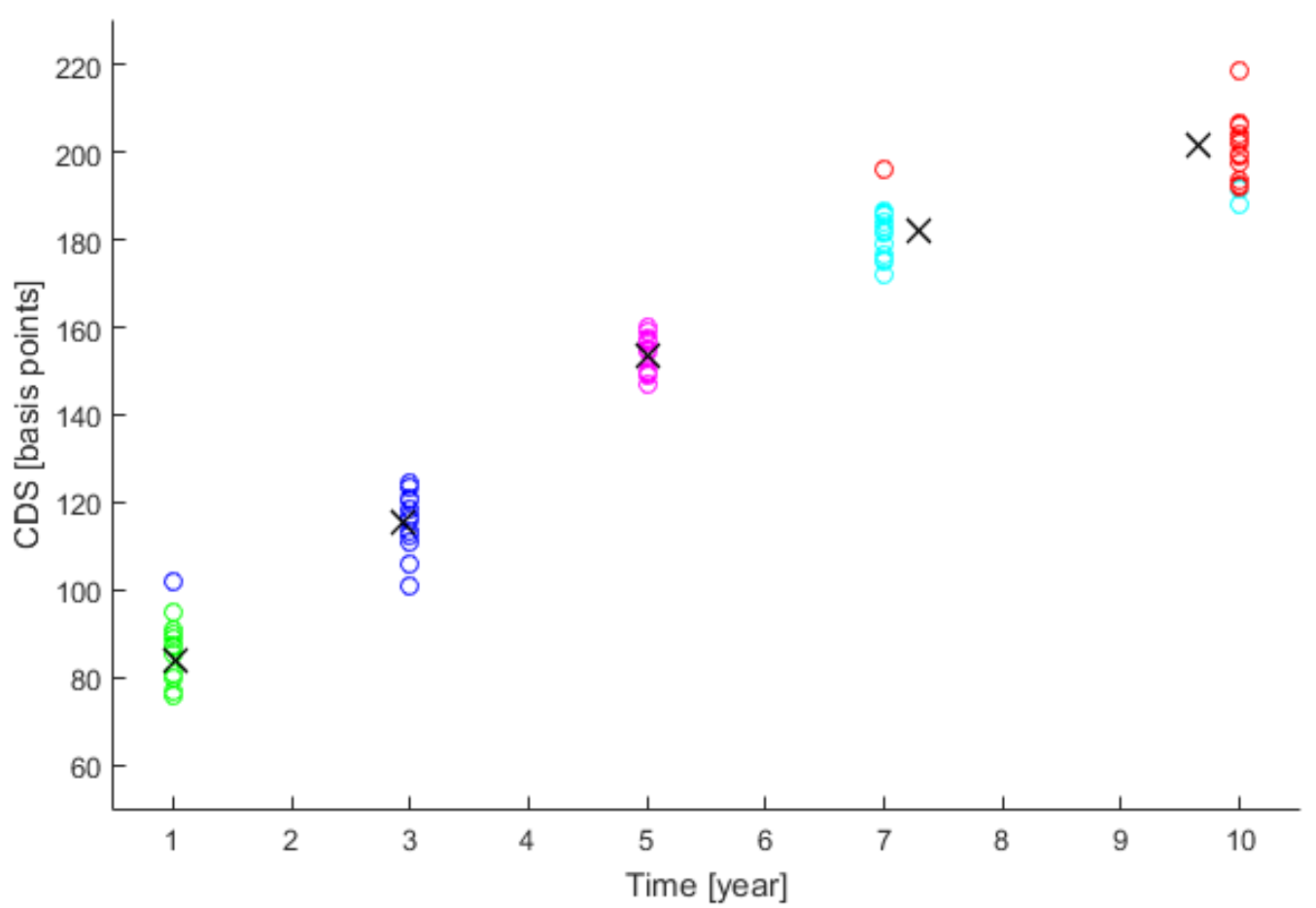

Figura 3: Fuzzy C-Means clustering del dataset e i relativi centri di massa

\begin{tabular}{|c|c|c|c|c|c|c|c|c|c|c|}
\hline Massa/ID & 1 & 2 & 3 & 4 & 5 & 6 & 7 & 8 & 9 & 10 \\
\hline 1,030 & $93,64 \%$ & $95,20 \%$ & $95,03 \%$ & $98,69 \%$ & $95,66 \%$ & $98,08 \%$ & $95,22 \%$ & $99,70 \%$ & $90,74 \%$ & $98,75 \%$ \\
\hline 2,934 & $4,24 \%$ & $3,24 \%$ & $3,35 \%$ & $0,92 \%$ & $3,33 \%$ & $1,34 \%$ & $3,23 \%$ & $0,22 \%$ & $7,27 \%$ & $0,94 \%$ \\
\hline 5,011 & $1,10 \%$ & $0,82 \%$ & $0,85 \%$ & $0,21 \%$ & $0,56 \%$ & $0,31 \%$ & $0,81 \%$ & $0,04 \%$ & $1,11 \%$ & $0,17 \%$ \\
\hline 7,291 & $0,59 \%$ & $0,43 \%$ & $0,45 \%$ & $0,11 \%$ & $0,27 \%$ & $0,16 \%$ & $0,43 \%$ & $0,02 \%$ & $0,52 \%$ & $0,08 \%$ \\
\hline 9,649 & $0,42 \%$ & $0,31 \%$ & $0,32 \%$ & $0,08 \%$ & $0,18 \%$ & $0,11 \%$ & $0,31 \%$ & $0,01 \%$ & $0,35 \%$ & $0,06 \%$ \\
\hline
\end{tabular}

\begin{tabular}{|c|c|c|c|c|c|c|c|c|c|c|}
\hline Massa/ID & 11 & 12 & 13 & 14 & 15 & 16 & 17 & 18 & 19 & 20 \\
\hline 1,030 & $73,70 \%$ & $33,50 \%$ & $93,81 \%$ & $97,93 \%$ & $2,65 \%$ & $38,56 \%$ & $0,96 \%$ & $14,47 \%$ & $0,22 \%$ & $0,21 \%$ \\
\hline 2,934 & $21,71 \%$ & $59,67 \%$ & $4,80 \%$ & $1,57 \%$ & $95,69 \%$ & $54,56 \%$ & $98,31 \%$ & $80,44 \%$ & $99,52 \%$ & $99,53 \%$ \\
\hline 5,011 & $2,62 \%$ & $4,05 \%$ & $0,77 \%$ & $0,28 \%$ & $1,05 \%$ & $4,06 \%$ & $0,47 \%$ & $3,11 \%$ & $0,17 \%$ & $0,17 \%$ \\
\hline 7,291 & $1,18 \%$ & $1,68 \%$ & $0,37 \%$ & $0,14 \%$ & $0,38 \%$ & $1,71 \%$ & $0,16 \%$ & $1,21 \%$ & $0,05 \%$ & $0,05 \%$ \\
\hline 9,649 & $0,79 \%$ & $1,09 \%$ & $0,25 \%$ & $0,09 \%$ & $0,23 \%$ & $1,11 \%$ & $0,10 \%$ & $0,77 \%$ & $0,03 \%$ & $0,03 \%$ \\
\hline
\end{tabular}




\begin{tabular}{|c|c|c|c|c|c|c|c|c|c|c|}
\hline Massa/ID & 21 & 22 & 23 & 24 & 25 & 26 & 27 & 28 & 29 & 30 \\
\hline 1,030 & $0,51 \%$ & $0,89 \%$ & $2,48 \%$ & $1,78 \%$ & $0,04 \%$ & $4,27 \%$ & $3,83 \%$ & $2,24 \%$ & $0,46 \%$ & $0,32 \%$ \\
\hline 2,934 & $99,07 \%$ & $97,81 \%$ & $95,94 \%$ & $95,14 \%$ & $99,91 \%$ & $84,22 \%$ & $86,69 \%$ & $93,54 \%$ & $1,70 \%$ & $1,16 \%$ \\
\hline 5,011 & $0,27 \%$ & $0,88 \%$ & $1,00 \%$ & $2,11 \%$ & $0,03 \%$ & $8,24 \%$ & $6,74 \%$ & $2,92 \%$ & $95,37 \%$ & $96,69 \%$ \\
\hline 7,291 & $0,09 \%$ & $0,27 \%$ & $0,36 \%$ & $0,61 \%$ & $0,01 \%$ & $2,10 \%$ & $1,76 \%$ & $0,83 \%$ & $1,77 \%$ & $1,32 \%$ \\
\hline 9,649 & $0,06 \%$ & $0,16 \%$ & $0,22 \%$ & $0,35 \%$ & $0,01 \%$ & $1,17 \%$ & $0,99 \%$ & $0,47 \%$ & $0,70 \%$ & $0,51 \%$ \\
\hline
\end{tabular}

\begin{tabular}{|c|c|c|c|c|c|c|c|c|c|c|}
\hline Massa/ID & 31 & 32 & 33 & 34 & 35 & 36 & 37 & 38 & 39 & 40 \\
\hline 1,030 & $0,93 \%$ & $0,24 \%$ & $0,41 \%$ & $0,04 \%$ & $0,25 \%$ & $0,42 \%$ & $0,46 \%$ & $0,16 \%$ & $0,03 \%$ & $0,19 \%$ \\
\hline 2,934 & $3,66 \%$ & $0,85 \%$ & $1,52 \%$ & $0,14 \%$ & $0,90 \%$ & $1,55 \%$ & $1,72 \%$ & $0,48 \%$ & $0,10 \%$ & $0,60 \%$ \\
\hline 5,011 & $91,11 \%$ & $97,50 \%$ & $95,80 \%$ & $99,43 \%$ & $97,37 \%$ & $95,74 \%$ & $95,33 \%$ & $97,71 \%$ & $99,61 \%$ & $97,10 \%$ \\
\hline 7,291 & $3,05 \%$ & $1,02 \%$ & $1,63 \%$ & $0,29 \%$ & $1,07 \%$ & $1,65 \%$ & $1,79 \%$ & $1,24 \%$ & $0,20 \%$ & $1,60 \%$ \\
\hline 9,649 & $1,25 \%$ & $0,39 \%$ & $0,64 \%$ & $0,10 \%$ & $0,41 \%$ & $0,65 \%$ & $0,70 \%$ & $0,40 \%$ & $0,07 \%$ & $0,51 \%$ \\
\hline
\end{tabular}

\begin{tabular}{|c|c|c|c|c|c|c|c|c|c|c|}
\hline Massa/ID & 41 & 42 & 43 & 44 & 45 & 46 & 47 & 48 & 49 & 50 \\
\hline 1,030 & $0,31 \%$ & $0,52 \%$ & $0,64 \%$ & $0,19 \%$ & $0,25 \%$ & $0,03 \%$ & $0,91 \%$ & $0,89 \%$ & $0,40 \%$ & $0,04 \%$ \\
\hline 2,934 & $0,94 \%$ & $1,54 \%$ & $1,84 \%$ & $0,60 \%$ & $0,75 \%$ & $0,08 \%$ & $2,19 \%$ & $2,14 \%$ & $0,93 \%$ & $0,09 \%$ \\
\hline 5,011 & $95,05 \%$ & $90,62 \%$ & $87,88 \%$ & $97,10 \%$ & $96,19 \%$ & $99,66 \%$ & $20,75 \%$ & $20,01 \%$ & $6,84 \%$ & $0,44 \%$ \\
\hline 7,291 & $2,84 \%$ & $5,68 \%$ & $7,53 \%$ & $1,60 \%$ & $2,14 \%$ & $0,17 \%$ & $68,19 \%$ & $69,06 \%$ & $86,75 \%$ & $98,14 \%$ \\
\hline 9,649 & $0,87 \%$ & $1,64 \%$ & $2,11 \%$ & $0,51 \%$ & $0,67 \%$ & $0,06 \%$ & $7,96 \%$ & $7,89 \%$ & $5,08 \%$ & $1,30 \%$ \\
\hline
\end{tabular}

\begin{tabular}{|c|c|c|c|c|c|c|c|c|c|c|}
\hline Massa/ID & 51 & 52 & 53 & 54 & 55 & 56 & 57 & 58 & 59 & 60 \\
\hline 1,030 & $0,04 \%$ & $0,10 \%$ & $0,31 \%$ & $0,49 \%$ & $0,00 \%$ & $0,00 \%$ & $0,15 \%$ & $0,25 \%$ & $0,24 \%$ & $0,12 \%$ \\
\hline 2,934 & $0,09 \%$ & $0,22 \%$ & $0,71 \%$ & $1,13 \%$ & $0,01 \%$ & $0,00 \%$ & $0,31 \%$ & $0,47 \%$ & $0,47 \%$ & $0,25 \%$ \\
\hline 5,011 & $0,44 \%$ & $1,34 \%$ & $5,04 \%$ & $8,66 \%$ & $0,05 \%$ & $0,01 \%$ & $1,46 \%$ & $1,71 \%$ & $1,71 \%$ & $1,19 \%$ \\
\hline 7,291 & $98,10 \%$ & $96,63 \%$ & $89,67 \%$ & $83,98 \%$ & $99,85 \%$ & $99,96 \%$ & $91,65 \%$ & $15,92 \%$ & $15,80 \%$ & $93,72 \%$ \\
\hline 9,649 & $1,33 \%$ & $1,71 \%$ & $4,27 \%$ & $5,75 \%$ & $0,09 \%$ & $0,02 \%$ & $6,42 \%$ & $81,65 \%$ & $81,77 \%$ & $4,73 \%$ \\
\hline
\end{tabular}

\begin{tabular}{|c|c|c|c|c|c|c|c|c|c|c|}
\hline Massa/ID & 61 & 62 & 63 & 64 & 65 & 66 & 67 & 68 & 69 & 70 \\
\hline 1,030 & $0,19 \%$ & $0,01 \%$ & $0,32 \%$ & $0,40 \%$ & $0,38 \%$ & $0,16 \%$ & $0,16 \%$ & $0,04 \%$ & $0,34 \%$ & $0,41 \%$ \\
\hline 2,934 & $0,40 \%$ & $0,03 \%$ & $0,65 \%$ & $0,79 \%$ & $0,74 \%$ & $0,29 \%$ & $0,29 \%$ & $0,07 \%$ & $0,67 \%$ & $0,81 \%$ \\
\hline 5,011 & $1,84 \%$ & $0,14 \%$ & $2,83 \%$ & $3,11 \%$ & $2,85 \%$ & $0,84 \%$ & $0,86 \%$ & $0,23 \%$ & $2,53 \%$ & $3,22 \%$ \\
\hline 7,291 & $88,39 \%$ & $99,45 \%$ & $76,68 \%$ & $43,58 \%$ & $35,75 \%$ & $3,93 \%$ & $3,99 \%$ & $1,59 \%$ & $28,97 \%$ & $48,49 \%$ \\
\hline 9,649 & $9,18 \%$ & $0,37 \%$ & $19,52 \%$ & $52,12 \%$ & $60,29 \%$ & $94,79 \%$ & $94,70 \%$ & $98,07 \%$ & $67,48 \%$ & $47,08 \%$ \\
\hline
\end{tabular}

\begin{tabular}{|c|c|c|c|c|c|c|c|}
\hline Massa/ID & 71 & 72 & 73 & 74 & 75 & 76 & 77 \\
\hline 1,030 & $0,10 \%$ & $0,00 \%$ & $0,02 \%$ & $1,21 \%$ & $0,13 \%$ & $0,04 \%$ & $0,03 \%$ \\
\hline 2,934 & $0,19 \%$ & $0,01 \%$ & $0,05 \%$ & $2,05 \%$ & $0,23 \%$ & $0,08 \%$ & $0,05 \%$ \\
\hline 5,011 & $0,65 \%$ & $0,02 \%$ & $0,14 \%$ & $5,16 \%$ & $0,69 \%$ & $0,25 \%$ & $0,18 \%$ \\
\hline 7,291 & $4,99 \%$ & $0,10 \%$ & $0,76 \%$ & $16,34 \%$ & $3,31 \%$ & $1,30 \%$ & $1,21 \%$ \\
\hline 9,649 & $94,07 \%$ & $99,88 \%$ & $99,03 \%$ & $75,23 \%$ & $95,63 \%$ & $98,32 \%$ & $98,53 \%$ \\
\hline
\end{tabular}

Tabella 3: Livelli di appartenenza dei punti del dataset ai singoli cluster

Il trader è in grado con questo metodo di visualizzare graficamente le eventuali anomalie di mercato: facendo riferimento all'output grafico di Figura 3, qualora non fossero presenti quotazioni anomale, i centri di massa (X) dovrebbero essere allineati al tenor di appartenenza della struttura a termine dei CDS. Maggiore è lo scostamento delle ascisse dei baricentri rispetto al tenor, maggiore è l'overlapping tra gli insiemi identificati e, conseguentemente, risulta più marcata l'anomalia. 
In un'ottica di algo-trading, la procedura può essere automatizzata: facendo riferimento alla matrice dei livelli di appartenenza, riportata nella Tabella 3, possono essere estratti i potenziali CDS anomali a partire dall'analisi delle percentuali di appartenenza ai singoli cluster per ogni punto.

Qualora la percentuale di appartenenza ad un gruppo fosse inferiore ad una determinata soglia limite di attenzione, il sistema informativo evidenzia l'anomalia all'operatore.

\section{Conclusioni}

L'articolo ha evidenziato come l'algoritmo Fuzzy C-Means clustering possa essere ritenuto idoneo a classificare le informazioni di mercato al fine di individuarne anomalie. La metodologia si presta ad essere implementata in una procedura automatica che sfrutta i livelli percentuali di appartenenza di un elemento rispetto alle partizioni individuate, oppure da interfaccia grafica con la visualizzazione degli scostamenti degli elementi rispetto ai baricentri calcolati.

Il principale aspetto negativo della metodologia, di particolare interesse nel settore finanziario, è che questa può operare laddove possono essere specificati a-priori il numero di cluster secondo il quale è ragionevole partizionare il set di dati in esame. Nell'esempio presentato era infatti ragionevole imporre come criterio la granularità temporale in corrispondenza della quale erano contribuiti i premi. E' intuitivo che l'algoritmo sia idoneo ad essere applicato in tutte le casistiche finanziarie che permettono di individuare un criterio per la suddivisione a-priori in classi dei dati (si cita ad esempio le strutture a termine contribuite di prezzi, tassi, volatilità, CDS, premi assicurativi).

Per il proseguo dello studio si ritiene interessante proporre una metodologia di partizione in grado, a fronte di un lieve incremento del tempo computazionale, di trattare problemi di clusterizzazione qualora il set iniziale di dati non consenta di specificare a monte un numero di classi all'interno delle quali suddividere il campione.

\section{Bibliografia}

- [1] J. F. Baldwin - "A new approach to approximate reasoning using Fuzzy Logic", FSS 2, 1979

- [2] J. C. Bezdec - "Pattern Recognition with Fuzzy Objective Function Algorithms", Plenum Press, New York, 1981

- [3] G. Bojadziev, M. Bojadziev - "Fuzzy Logic for Business, Finance and Management" - Advances in Fuzzy Systems: Applications and Theory, Vol. 23, 2007

- [4] S. Chiu - "Fuzzy Model Identification based on Cluster Estimation", Journal of Intelligent \& Fuzzy Systems, Vol. 2, No. 3, 1994

- [5] D. E. Cox - "Fuzzy Logic for Business and Industry", Charles River Media Inc., Rockland, Massachusetts, 1995

- [6] A. M. Gil Lafuente - "Fuzzy Logic in Financial Analysis" - Springer series in "Fuzziness and Soft Computing", Vol. 175, 2005

- [7] J. Harris - "Fuzzy Logic Applications in Engineering Science", Springer series in "Microprocessor based and intelligent systems engineering", Vol. 29, 2006

- [8] H. Hellendoorn, C. Thomas - "Defuzzification Fuzzy Controllers", Journal of Intelligent and Fuzzy System, 1993

- [9] A. Kaufmann - "Introduction to the Theory of Fuzzy Subsets", Academic Press, New York, 1975

- [10] A. Kaufmann, M. M. Gupta - "Fuzzy Mathematical Models in Engineering and Management Sciences", NorthHolland, Amsterdam, 1988

- [11] T. Korol - "Fuzzy Logic - Emerging Technologies and Applications: Fuzzy Logic in Financial Management" InTech Pubblication, 2012

- $\quad$ [12] B. Kosko - "Fuzzy Thinking: The New Science of Fuzzy Logic", Hyperion, 1993

- [13] A. M. Ribeiro, L. B. Neto, P. H. G. Coelho, J. C. Soares de Mello, L. A. Meza - "Using Fuzzy Logic for pricing" - ICEIS 2005 - Artificial Intelligence and Decision Support Systems, 2005

- [14] G. Stojic - "Using Fuzzy Logic for evaluating the level of countries' (regions') Economic Development" Panoeconomicus, 2012

- [15] The Mathworks - "Fuzzy Logic Toolbox" - User’s Guide, 2015

- [16] The Mathworks - "Statistics and Machine Learning Toolbox" - User’s Guide, 2015

- [17] L. Zadeh - "Fuzzy sets", Information and Control. 1965 
Risk Management Magazine

Anno 12, $\mathrm{n}^{\circ} 1$ Gennaio - Aprile 2016

Direttore Responsabile

Maurizio Vallino

Condirettore

Corrado Meglio

Consiglio Scientifico

Giampaolo Gabbi (Direttore del Consiglio Scientifico),

AnnaBottasso,

Ruggero Bertelli,

Ottavio Caligaris,

Simona Cosma,

Paola Ferretti,

Andrea Giacomelli,

Adele Grassi,

Pier Giuseppe Giribone,

Duccio Martelli,

Anna Grazia Quaranta,

Enzo Scannella,

Cristiana Schena,

Giuseppe Torluccio

Comitato di redazione

Emanuele Diquattro

Fausto Galmarini

Rossano Giuppa

Aldo Letizia

Paolo Palliola

Enzo Rocca

Fabio Salis

Vignettista: Silvano Gaggero

Proprietà, Redazione e Segreteria:

Associazione Italiana Financial Industry Risk Managers (AIFIRM), Via Sile 18, 20139 Milano

Registrazione del Tribunale di Milano $\mathrm{n}^{\circ} 629$ del 10/9/2004

E-mail: segreteria@ aifirm.it;

Tel. 3896946315

Lunedì - Venerdì h.15-17

Stampa: Algraphy S.n.c. - Passo Ponte Carrega 62-62r, 16141 Genova

Le opinioni espresse negli articoli impegnano unicamente la responsabilità dei rispettivi autori

SPEDIZIONE IN ABBONAMENTO POSTALE AI SOCI AIFIRM RESIDENTI IN ITALIA, IN REGOLA CON L'ISCRIZIONE 


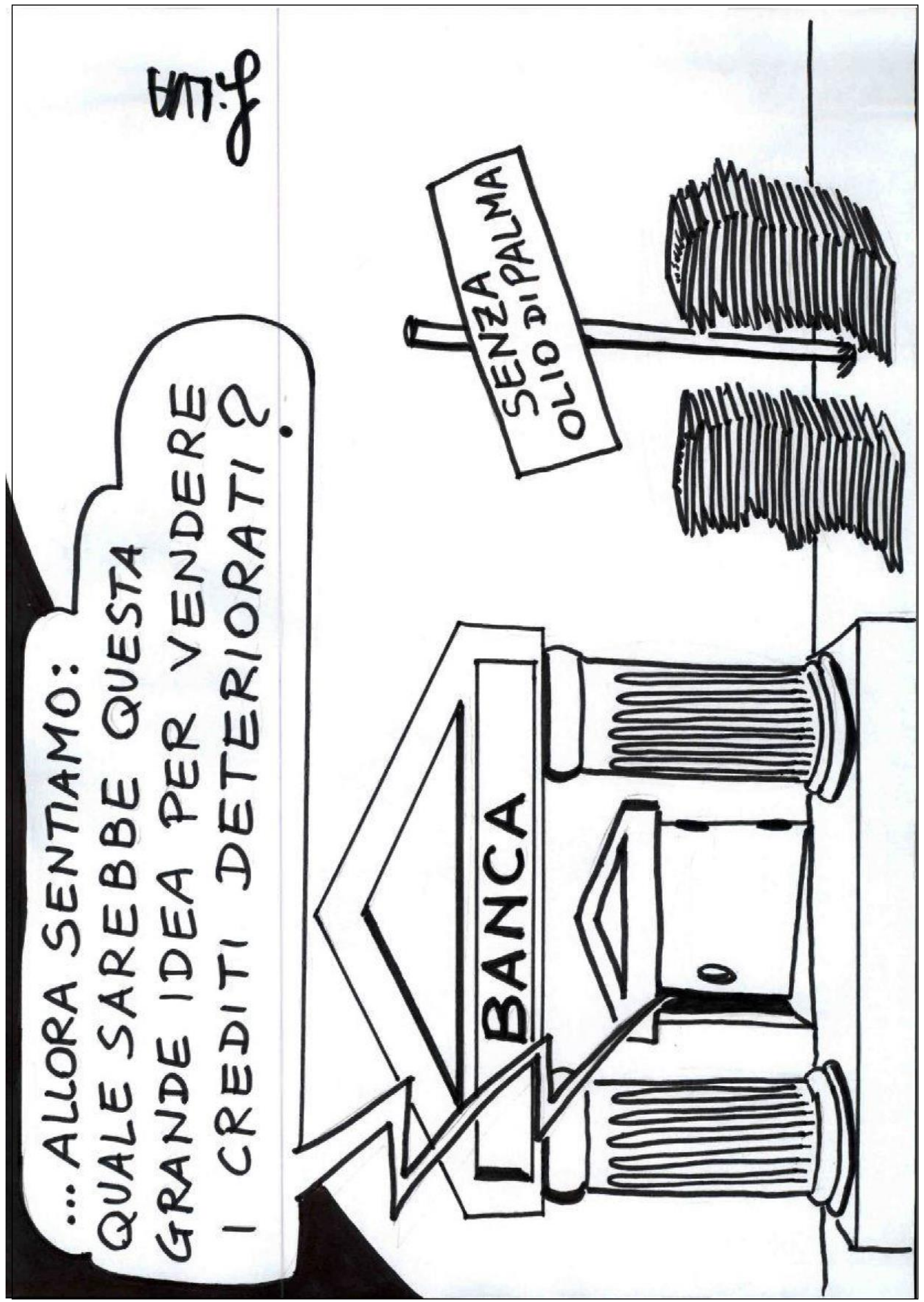

NEWSLETTER AIFIRM RISK MANAGEMENT MAGAZINE ANNO 12 N 1 - PAGINA - 35 - 


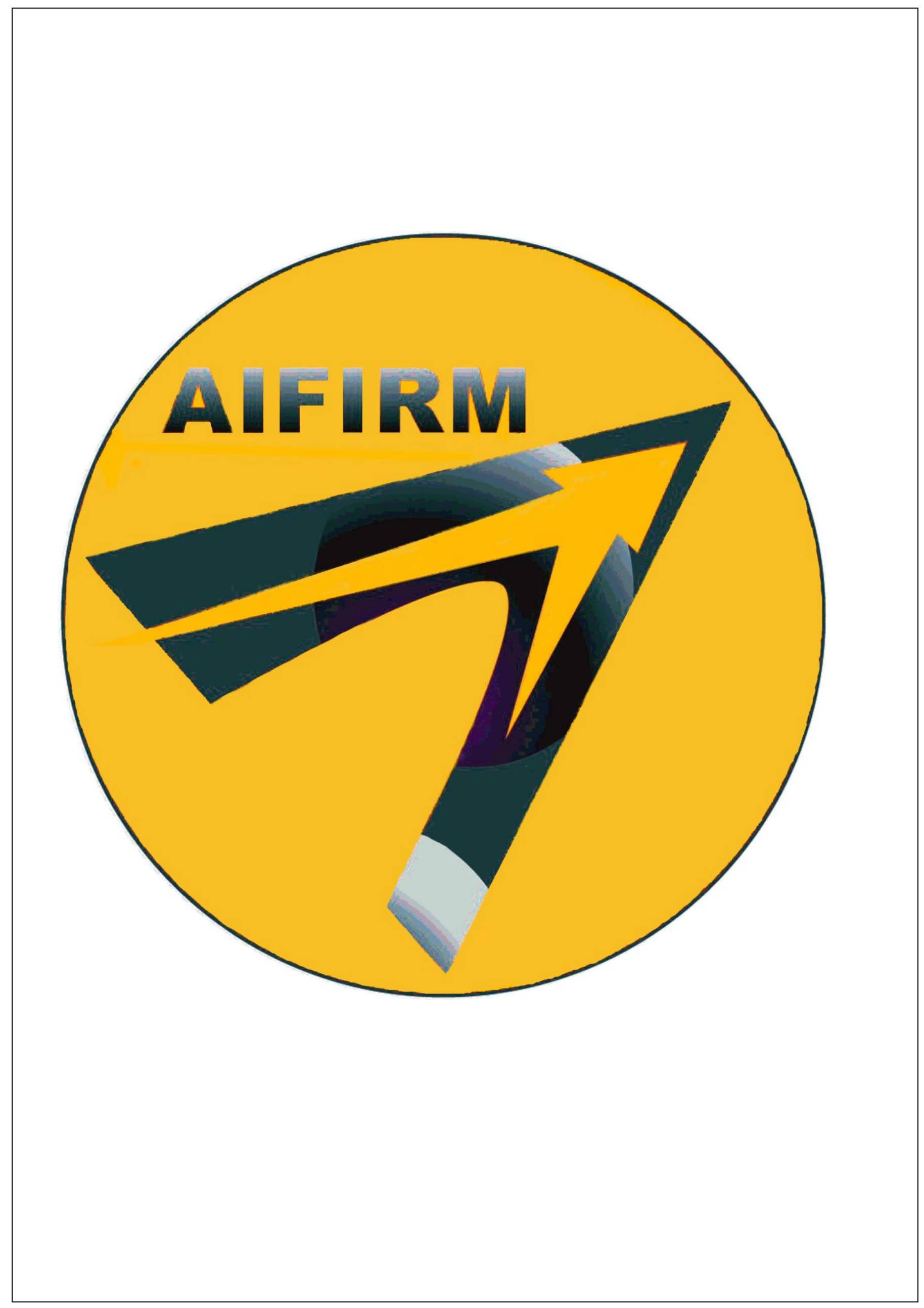

NEWSLETTER AIFIRM RISK MANAGEMENT MAGAZINE ANNO 12 N 1 - PAGINA - 36 - 